\title{
Scale hierarchies in particle physics and cosmology
}

\author{
I. Antoniadis ${ }^{1,2, a}$ \\ ${ }^{1}$ LPTHE, UMR CNRS 7589 Sorbonne Universités, UPMC Paris 6, 75005 Paris, France \\ ${ }^{2}$ Albert Einstein Center, Institute for Theoretical Physics, Bern University, Sidlerstrasse 5, CH-3012 Bern, \\ Switzerland
}

\begin{abstract}
I describe the phenomenology of a model of supersymmetry breaking in the presence of a tiny (tuneable) positive cosmological constant. It utilises a single chiral multiplet with a gauged shift symmetry, that can be identified with the string dilaton (or an appropriate compactification modulus). The model is coupled to the MSSM, leading to calculable soft supersymmetry breaking masses and a distinct low energy phenomenology that allows to differentiate it from other models of supersymmetry breaking and mediation mechanisms. We also study the question if this model can lead to inflation by identifying the dilaton with the inflaton. We find that this is possible if the Kähler potential is modified by a term that has the form of NS5-brane instantons, leading to an appropriate inflationary plateau around the maximum of the scalar potential, depending on two extra parameters.
\end{abstract}

\section{Introduction}

If String Theory is a fundamental theory of Nature and not just a tool for studying systems with strongly coupled dynamics, it should be able to describe at the same time particle physics and cosmology, which are phenomena that involve very different scales from the microscopic four-dimensional (4d) quantum gravity length of $10^{-33} \mathrm{~cm}$ to large macroscopic distances of the size of the observable Universe $\sim 10^{28} \mathrm{~cm}$ spanned a region of about 60 orders of magnitude. In particular, besides the $4 \mathrm{~d}$ Planck mass, there are three very different scales with very different physics corresponding to the electroweak, dark energy and inflation. These scales might be related via the scale of the underlying fundamental theory, such as string theory, or they might be independent in the sense that their origin could be based on different and independent dynamics. An example of the former constraint and more predictive possibility is provided by $\mathrm{TeV}$ strings with a fundamental scale at low energies due for instance to large extra dimensions transverse to a four-dimensional braneworld forming our Universe [1]. In this case, the $4 \mathrm{~d}$ Planck mass is emergent from the fundamental string scale and inflation should also happen around the same scale [2].

Here, we will adopt the second more conservative approach, assuming that all three scales have an independent dynamical origin. Moreover, we will assume the presence of low energy supersymmetry that allows for an elegant solution of the mass hierarchy problem, a unification of fundamental forces as indicated by low energy data and a natural dark matter candidate due to an unbroken R-parity.

\footnotetext{
ae-mail: ignatios.antoniadis@upmc.fr
} 
The assumption of independent scales implies that supersymmetry breaking should be realized in a metastable de Sitter vacuum with an infinitesimally small (tunable) cosmological constant independent of the supersymmetry breaking scale that should be in the TeV region. In a recent work [3], we studied a simple $N=1$ supergravity model having this property and motivated by string theory. Besides the gravity multiplet, the minimal field content consists of a chiral multiplet with a shift symmetry promoted to a gauged R-symmetry using a vector multiplet. In the string theory context, the chiral multiplet can be identified with the string dilaton (or an appropriate compactification modulus) and the shift symmetry associated to the gauge invariance of a two-index antisymmetric tensor that can be dualized to a (pseudo)scalar. The shift symmetry fixes the form of the superpotential and the gauging allows for the presence of a Fayet-Iliopoulos (FI) term, leading to a supergravity action with two independent parameters that can be tuned so that the scalar potential possesses a metastable de Sitter minimum with a tiny vacuum energy (essentially the relative strength between the F- and D-term contributions). A third parameter fixes the Vacuum Expectation Value (VEV) of the string dilaton at the desired (phenomenologically) weak coupling regime. An important consistency constraint of our model is anomaly cancellation which has been studied in [5] and implies the existence of additional charged fields under the gauged R-symmetry.

In a more recent work [6], we analyzed a small variation of this model which is manifestly anomaly free without additional charged fields and allows to couple in a straight forward way a visible sector containing the minimal supersymmetric extension of the Standard Model (MSSM) and studied the mediation of supersymmetry breaking and its phenomenological consequences. It turns out that an additional 'hidden sector' field $z$ is needed to be added for the matter soft scalar masses to be nontachyonic; although this field participates in the supersymmetry breaking and is similar to the so-called Polonyi field, it does not modify the main properties of the metastable de Sitter (dS) vacuum. All soft scalar masses, as well as trilinear A-terms, are generated at the tree level and are universal under the assumption that matter kinetic terms are independent of the 'Polonyi' field, since matter fields are neutral under the shift symmetry and supersymmetry breaking is driven by a combination of the $U(1)$ D-term and the dilaton and $z$-field F-term. Alternatively, a way to avoid the tachyonic scalar masses without adding the extra field $z$ is to modify the matter kinetic terms by a dilaton dependent factor.

A main difference of the second analysis from the first work is that we use a field representation in which the gauged shift symmetry corresponds to an ordinary $U(1)$ and not an R-symmetry. The two representations differ by a Kähler transformation that leaves the classical supergravity action invariant. However, at the quantum level, there is a Green-Schwarz term generated that amounts an extra dilaton dependent contribution to the gauge kinetic terms needed to cancel the anomalies of the R-symmetry. This creates an apparent puzzle with the gaugino masses that vanish in the first representation but not in the latter. The resolution to the puzzle is based to the so called anomaly mediation contributions $[7,8]$ that explain precisely the above apparent discrepancy. It turns out that gaugino masses are generated at the quantum level and are thus suppressed compared to the scalar masses (and A-terms).

This model has the necessary ingredients to be obtained as a remnant of moduli stabilisation within the framework of internal magnetic fluxes in type I string theory, turned on along the compact directions for several abelian factors of the gauge group. All geometric moduli can in principle be fixed in a supersymmetric way, while the shift symmetry is associated to the $4 \mathrm{~d}$ axion and its gauging is a consequence of anomaly cancellation $[9,10]$.

We then make an attempt to connect the scale of inflation with the electroweak and supersymmetry breaking scales within the same effective field theory, that at the same time allows the existence of an infinitesimally small (tuneable) positive cosmological constant describing the present dark energy of the universe. We thus address the question whether the same scalar potential can provide inflation 
with the dilaton playing also the role of the inflaton at an earlier stage of the universe evolution [11]. We show that this is possible if one modifies the Kähler potential by a correction that plays no role around the minimum, but creates an appropriate plateau around the maximum. In general, the Kähler potential receives perturbative and non-perturbative corrections that vanish in the weak coupling limit. After analysing all such corrections, we find that only those that have the form of (Neveu-Schwarz) NS5-brane instantons can lead to an inflationary period compatible with cosmological observations. The scale of inflation turns out then to be of the order of low energy supersymmetry breaking, in the $\mathrm{TeV}$ region. On the other hand, the predicted tensor-to-scalar ratio is too small to be observed.

Inflationary models [12] in supergravity ${ }^{1}$ suffer in general from several problems, such as finetuning to satisfy the slow-roll conditions, large field initial conditions that break the validity of the effective field theory, and stabilisation of the (pseudo) scalar companion of the inflaton arising from the fact that bosonic components of superfields are always even. The simplest argument to see the fine tuning of the potential is that a canonically normalised kinetic term of a complex scalar field $X$ corresponds to a quadratic Kähler potential $K=X \bar{X}$ that brings one unit contribution to the slow-roll parameter $\eta=V^{\prime \prime} / V$, arising from the $e^{K}$ proportionality factor in the expression of the scalar potential $V$. This problem can be avoided in models with no-scale structure where cancellations arise naturally due to non-canonical kinetic terms leading to potentials with flat directions (at the classical level). However, such models require often trans-Planckian initial conditions that invalidate the effective supergravity description during inflation. A concrete example where all these problems appear is the Starobinsky model of inflation [14], despite its phenomenological success.

All three problems above are solved when the inflaton is identified with the scalar component of the goldstino superfield ${ }^{2}$, in the presence of a gauged R-symmetry [16]. Indeed, the superpotential is in that case linear and the big contribution to $\eta$ described above cancels exactly. Since inflation arises in a plateau around the maximum of the scalar potential (hill-top) no large field initial conditions are needed, while the pseudo-scalar companion of the inflaton is absorbed into the R-gauge field that becomes massive, leading the inflaton as a single scalar field present in the spectrum. This model provides therefore a minimal realisation of natural small-field inflation in supergravity, compatible with present observations, as we show below. Moreover, it allows the presence of a realistic minimum describing our present Universe with an infinitesimal positive vacuum energy arising due to a cancellation between an F- and D-term contributions to the scalar potential, without affecting the properties of the inflationary plateau, along the lines of Ref. [3, 4, 11].

On general grounds, there are two classes of such models depending on whether the maximum corresponds to a point of unbroken (case 1) or broken (case 2) R-symmetry. The latter corresponds actually to a generalisation of the model we discussed above [11], inspired by string theory [3]. It has the same field content but in a different field basis with a chiral multiplet $S \propto \ln X$ playing the role of the string dilaton. Thus, $S$ has a shift symmetry which is actually an R-symmetry gauged by a vector multiplet and the superpotential is a single exponential. The scalar potential has a minimum with a tuneable vacuum energy and a maximum that can produce inflation when appropriate corrections are included in the Kähler potential. In these coordinates R-symmetry is restored at infinity, corresponding to the weak coupling limit. Small field inflation is again guaranteed consistently with the validity of the effective field theory.

\footnotetext{
${ }^{1}$ For reviews on supersymmetric models of inflation, see for example [13].

${ }^{2}$ See [15] for earlier work relating supersymmetry and inflation.
} 


\section{Conventions}

Throughout this paper we use the conventions of [17]. A supergravity theory is specified (up to ChernSimons terms) by a Kähler potential $\mathcal{K}$, a superpotential $W$, and the gauge kinetic functions $f_{A B}(z)$. The chiral multiplets $z^{\alpha}, \chi^{\alpha}$ are enumerated by the index $\alpha$ and the indices $A, B$ indicate the different gauge groups. Classically, a supergravity theory is invariant under Kähler tranformations, viz.

$$
\begin{aligned}
\mathcal{K}(z, \bar{z}) & \longrightarrow \mathcal{K}(z, \bar{z})+J(z)+\bar{J}(\bar{z}), \\
W(z) & \longrightarrow e^{-\kappa^{2} J(z)} W(z),
\end{aligned}
$$

where $\kappa$ is the inverse of the reduced Planck mass, $m_{p}=\kappa^{-1}=2.4 \times 10^{15} \mathrm{TeV}$. The gauge transformations of chiral multiplet scalars are given by holomorphic Killing vectors, i.e. $\delta z^{\alpha}=\theta^{A} k_{A}^{\alpha}(z)$, where $\theta^{A}$ is the gauge parameter of the gauge group $A$. The Kähler potential and superpotential need not be invariant under this gauge transformation, but can change by a Kähler transformation

$$
\delta \mathcal{K}=\theta^{A}\left[r_{A}(z)+\bar{r}_{A}(\bar{z})\right],
$$

provided that the gauge transformation of the superpotential satisfies $\delta W=-\theta^{A} \kappa^{2} r_{A}(z) W$. One then has from $\delta W=W_{\alpha} \delta z^{\alpha}$

$$
W_{\alpha} k_{A}^{\alpha}=-\kappa^{2} r_{A} W
$$

where $W_{\alpha}=\partial_{\alpha} W$ and $\alpha$ labels the chiral multiplets. The supergravity theory can then be described by a gauge invariant function

$$
\mathcal{G}=\kappa^{2} \mathcal{K}+\log \left(\kappa^{6} W \bar{W}\right)
$$

The scalar potential is given by

$$
\begin{aligned}
V & =V_{F}+V_{D} \\
V_{F} & =e^{\kappa^{2} \mathcal{K}}\left(-3 \kappa^{2} W \bar{W}+\nabla_{\alpha} W g^{\alpha \bar{\beta}} \bar{\nabla}_{\bar{\beta}} \bar{W}\right) \\
V_{D} & =\frac{1}{2}(\operatorname{Re} f)^{-1 A B} \mathcal{P}_{A} \mathcal{P}_{B},
\end{aligned}
$$

where W appears with its Kähler covariant derivative

$$
\nabla_{\alpha} W=\partial_{\alpha} W(z)+\kappa^{2}\left(\partial_{\alpha} \mathcal{K}\right) W(z)
$$

The moment maps $\mathcal{P}_{A}$ are given by

$$
\mathcal{P}_{A}=i\left(k_{A}^{\alpha} \partial_{\alpha} \mathcal{K}-r_{A}\right)
$$

In this paper we will be concerned with theories having a gauged R-symmetry, for which $r_{A}(z)$ is given by an imaginary constant $r_{A}(z)=i \kappa^{-2} \xi$. In this case, $\kappa^{-2} \xi$ is a Fayet-Iliopoulos [18] constant parameter.

\section{The model}

The starting point is a chiral multiplet $S$ and a vector multiplet associated with a shift symmetry of the scalar component $s$ of the chiral multiplet $S$

$$
\delta s=-i c \theta,
$$


and a string-inspired Kähler potential of the form $-p \log (s+\bar{s})$. The most general superpotential is either a constant $W=\kappa^{-3} a$ or an exponential superpotential $W=\kappa^{-3} a e^{b s}$ (where $a$ and $b$ are constants). A constant superpotential is (obviously) invariant under the shift symmetry, while an exponential superpotential transforms as $W \rightarrow W e^{-i b c \theta}$, as in eq. (3). In this case the shift symmetry becomes a gauged R-symmetry and the scalar potential contains a Fayet-Iliopoulos term. Note however that by performing a Kähler transformation (1) with $J=\kappa^{-2} b s$, the model can be recast into a constant superpotential at the cost of introducing a linear term in the Kähler potential $\delta K=b(s+\bar{s})$. Even though in this representation, the shift symmetry is not an R-symmetry, we will still refer to it as $U(1)_{R}$. The most general gauge kinetic function has a constant term and a term linear in $s, f(s)=\delta+\beta s$.

To summarise, ${ }^{3}$

$$
\begin{aligned}
\mathcal{K}(s, \bar{s}) & =-p \log (s+\bar{s})+b(s+\bar{s}), \\
W(s) & =a, \\
f(s) & =\delta+\beta s,
\end{aligned}
$$

where we have set the mass units $\kappa=1$. The constants $a$ and $b$ together with the constant $c$ in eq. (8) can be tuned to allow for an infinitesimally small cosmological constant and a TeV gravitino mass. For $b>0$, there always exists a supersymmetric AdS (anti-de Sitter) vacuum at $\langle s+\bar{s}\rangle=b / p$, while for $b=0$ (and $p<3$ ) there is an AdS vacuum with broken supersymmetry. We therefore focus on $b<0$. In the context of string theory, $S$ can be identified with a compactification modulus or the universal dilaton and (for negative $b$ ) the exponential superpotential may be generated by nonperturbative effects.

The scalar potential is given by:

$$
\begin{aligned}
V & =V_{F}+V_{D} \\
V_{F} & =a^{2} e^{\frac{b}{l}} l^{p-2}\left\{\frac{1}{p}(p l-b)^{2}-3 l^{2}\right\} \quad l=1 /(s+\bar{s}) \\
V_{D} & =c^{2} \frac{l}{\beta+2 \delta l}(p l-b)^{2}
\end{aligned}
$$

In the case where $S$ is the string dilaton, $V_{D}$ can be identified as the contribution of a magnetized D-brane, while $V_{F}$ for $b=0$ and $p=2$ coincides with the tree-level dilaton potential obtained by considering string theory away its critical dimension [19]. For $p \geq 3$ the scalar potential $V$ is positive and monotonically decreasing, while for $p<3$, its F-term part $V_{F}$ is unbounded from below when $s+\bar{s} \rightarrow 0$. On the other hand, the D-term part of the scalar potential $V_{D}$ is positive and diverges when $s+\bar{s} \rightarrow 0$ and for various values for the parameters an (infinitesimally small) positive (local) minimum of the potential can be found.

If we restrict ourselves to integer $p$, tunability of the vacuum energy restricts $p=2$ or $p=1$ when $f(s)=s$, or $p=1$ when the gauge kinetic function is constant. For $p=2$ and $f(s)=s$, the minimization of $V$ yields:

$$
\begin{aligned}
b / l & =-\rho_{0} \approx-0.183268 \quad, \quad p=2 \\
\frac{a^{2}}{b c^{2}} & =A_{2}\left(-\rho_{0}\right)+B_{2}\left(-\rho_{0}\right) \frac{\Lambda}{b^{3} c^{2}} \approx-50.6602+O(\Lambda),
\end{aligned}
$$

where $\Lambda$ is the value of $V$ at the minimum (i.e. the cosmological constant), $-\rho_{0}$ is the negative root of the polynomial $-x^{5}+7 x^{4}-10 x^{3}-22 x^{2}+40 x+8$ compatible with (12) for $\Lambda=0$ and $A_{2}(\alpha), B_{2}(\alpha)$

\footnotetext{
${ }^{3}$ In superfields the shift symmetry (8) is given by $\delta S=-i c \Lambda$, where $\Lambda$ is the superfield generalization of the gauge parameter. The gauge invariant Kähler potential is then given by $\mathcal{K}(S, \bar{S})=-p \kappa^{-2} \log \left(S+\bar{S}+c V_{R}\right)+\kappa^{-2} b\left(S+\bar{S}+c V_{R}\right)$, where $V_{R}$ is the gauge superfield of the shift symmetry.
} 
are given by

$$
A_{2}(\alpha)=2 e^{-\alpha} \frac{-4+4 \alpha-\alpha^{2}}{\alpha^{3}-4 \alpha^{2}-2 \alpha} \quad ; \quad B_{2}(\alpha)=2 \frac{\alpha^{2} e^{-\alpha}}{\alpha^{2}-4 \alpha-2}
$$

It follows that by carefully tuning $a$ and $c, \Lambda$ can be made positive and arbitrarily small independently of the supersymmetry breaking scale. A plot of the scalar potential for certain values of the parameters is shown in figure 1 .

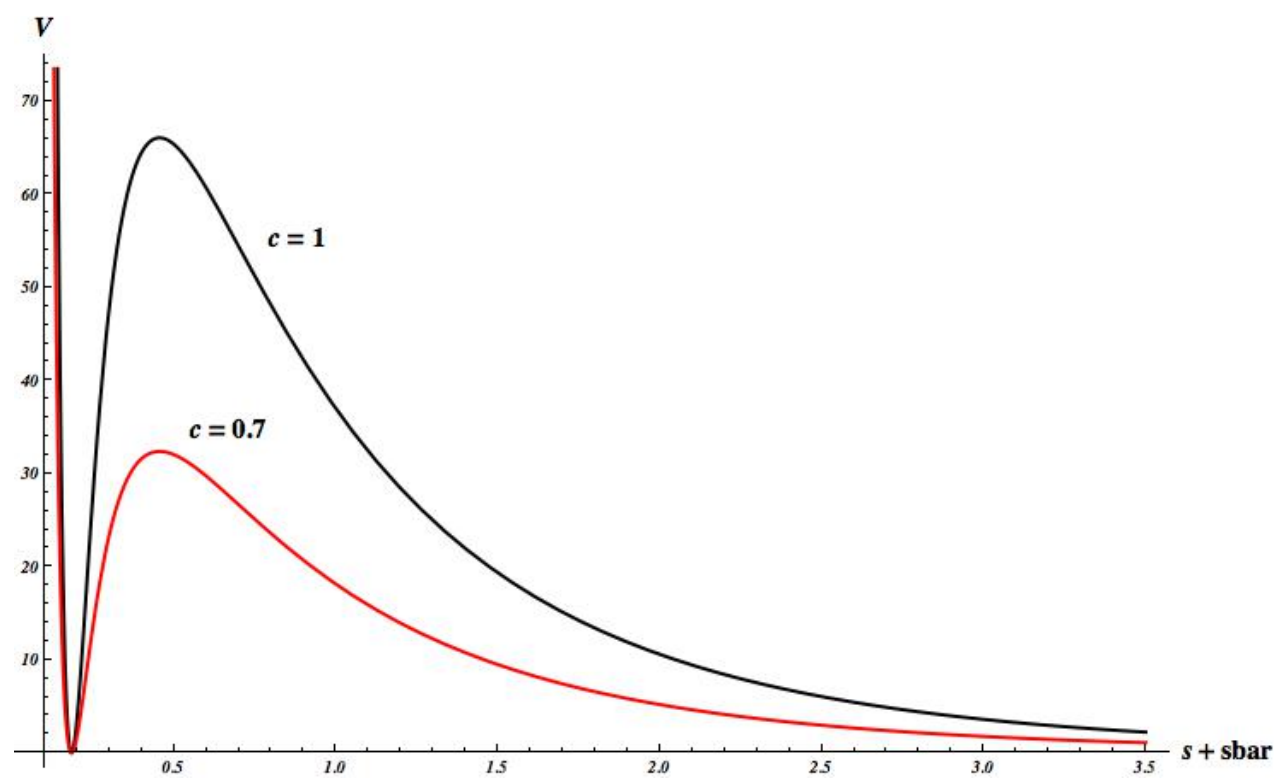

Figure 1. A plot of the scalar potential for $p=2, b=-1, \delta=0, \beta=1$ and $a$ given by equation (12) for $c=1$ (black curve) and $c=0.7$ (red curve).

At the minimum of the scalar potential, for nonzero $a$ and $b<0$, supersymmetry is broken by expectation values of both an F and D-term. Indeed the F-term and D-term contributions to the scalar potential are

$$
\begin{aligned}
& \left.V_{F}\right|_{s+\bar{s}=\frac{-\rho_{0}}{b}}=\frac{1}{2} a^{2} b^{2} e^{-} \rho_{0}\left(1+\frac{2}{\rho_{0}}\right)^{2}>0, \\
& \left.V_{D}\right|_{s+\bar{s}=\frac{-\rho_{0}}{b}}=-\frac{b^{3} c^{2}}{\rho_{0}}\left(1+\frac{2}{\rho_{0}}\right)^{2}>0 .
\end{aligned}
$$

The gravitino mass term is given by

$$
\left(m_{3 / 2}\right)^{2}=e^{\mathcal{G}}=\frac{a^{2} b^{2}}{\rho_{0}^{2}} e^{-\rho_{0}} .
$$

Due to the Stueckelberg coupling, the imaginary part of $s$ (the axion) gets eaten by the gauge field, which acquires a mass. On the other hand, the Goldstino, which is a linear combination of the fermion of the chiral multiplet $\chi$ and the gaugino $\lambda$ gets eaten by the gravitino. As a result, the physical spectrum of the theory consists (besides the graviton) of a massive scalar, namely the dilaton, a Majorana 
fermion, a massive gauge field and a massive gravitino. All the masses are of the same order of magnitude as the gravitino mass, proportional to the same constant $a$ (or $c$ related by eq. (12) where $b$ is fixed by eq. (11)), which is a free parameter of the model. Thus, they vanish in the same way in the supersymmetric limit $a \rightarrow 0$.

The local $\mathrm{dS}$ minimum is metastable since it can tunnel to the supersymmetric ground state at infinity in the $s$-field space (zero coupling). It turns out however that it is extremely long lived for realistic perturbative values of the gauge coupling $l \simeq 0.02$ and $\mathrm{TeV}$ gravitino mass and, thus, practically stable; its decay rate is [5]:

$$
\Gamma \sim e^{-B} \quad \text { with } \quad B \approx 10^{300} .
$$

\section{Coupling a visible sector}

The guideline to construct a realistic model keeping the properties of the toy model described above is to assume that matter fields are invariant under the shift symmetry (8) and do not participate in the supersymmetry breaking. In the simplest case of a canonical Kähler potential, MSSM-like fields $\phi$ can then be added as:

$$
\begin{aligned}
\mathcal{K} & =-\kappa^{-2} \log (s+\bar{s})+\kappa^{-2} b(s+\bar{s})+\sum \varphi \bar{\varphi}, \\
W & =\kappa^{-3} a+W_{M S S M},
\end{aligned}
$$

where $W_{M S S M}(\phi)$ is the usual MSSM superpotential. The squared soft scalar masses of such a model can be shown to be positive and close to the square of the gravitino mass $\left(\mathrm{TeV}^{2}\right)$. On the other hand, for a gauge kinetic function with a linear term in $s, \beta \neq 0$ in eq. (9), the Lagrangian is not invariant under the shift symmetry

$$
\delta \mathcal{L}=-\theta \frac{\beta c}{8} \epsilon^{\mu \nu \rho \sigma} F_{\mu \nu} F_{\rho \sigma} .
$$

and its variation should be canceled. As explained in Ref. [5], in the 'frame' with an exponential superpotential the R-charges of the fermions in the model can give an anomalous contribution to the Lagrangian. In this case the 'Green-Schwarz' term $\operatorname{Im} s F \tilde{F}$ can cancel quantum anomalies. However as shown in [5], with the minimal MSSM spectrum, the presence of this term requires the existence of additional fields in the theory charged under the shift symmetry.

Instead, to avoid the discussion of anomalies, we focus on models with a constant gauge kinetic function. In this case the only (integer) possibility ${ }^{4}$ is $p=1$. The scalar potential is given by (10) with $\beta=0, \delta=p=1$. The minimization yields to equations similar to (11), (12) and (13) with a different value of $\rho_{0}$ and functions $A_{1}$ and $B_{1}$ given by:

$$
\begin{aligned}
b\langle s+\bar{s}\rangle & =-\rho_{0} \approx-0.233153 \\
\frac{b c^{2}}{a^{2}} & =A_{1}\left(-\rho_{0}\right)+B_{1}\left(-\rho_{0}\right) \frac{\Lambda}{a^{2} b} \approx-0.359291+O(\Lambda) \\
A_{1}(\alpha) & =2 e^{\alpha} \alpha \frac{3-(\alpha-1)^{2}}{(\alpha-1)^{2}} \quad, \quad B_{1}(\alpha)=\frac{2 \alpha^{2}}{(\alpha-1)^{2}},
\end{aligned}
$$

where $-\rho_{0}$ is the negative root of $-3+(\rho-1)^{2}\left(2-\rho^{2} / 2\right)=0$ close to -0.23 , compatible with the second constraint for $\Lambda=0$. However, this model suffers from tachyonic soft masses when it is coupled to

\footnotetext{
${ }^{4}$ If $f(s)$ is constant, the leading contribution to $V_{D}$ when $s+\bar{s} \rightarrow 0$ is proportional to $1 /(s+\bar{s})^{2}$, while the leading contribution to $V_{F}$ is proportional to $1 /(s+\bar{s})^{p}$. It follows that $p<2$; if $p>2$, the potential is unbounded from below, while if $p=2$, the potential is either positive and monotonically decreasing or unbounded from below when $s+\bar{s} \rightarrow 0$ depending on the values of the parameters.
} 
the MSSM, as in (17). To circumvent this problem, one can add an extra hidden sector field which contributes to (F-term) supersymmetry breaking. Alternatively, the problem of tachyonic soft masses can also be solved if one allows for a non-canonical Kähler potential in the visible sector, which gives an additional contribution to the masses through the D-term.

Let us discuss first the addition of an extra hidden sector field $z$ (similar to the so-called Polonyi field [20]). The Kähler potential, superpotential and gauge kinetic function are given by

$$
\begin{aligned}
\mathcal{K} & =-\kappa^{-2} \log (s+\bar{s})+\kappa^{-2} b(s+\bar{s})+z \bar{z}+\sum \varphi \bar{\varphi}, \\
W & =\kappa^{-3} a(1+\gamma \kappa z)+W_{\mathrm{MSSM}}(\varphi), \\
f(s) & =1, \quad f_{A}=1 / g_{A}^{2},
\end{aligned}
$$

where $A$ labels the Standard Model gauge group factors and $\gamma$ is an additional constant parameter. The existence of a tuneable dS vacuum with supersymmetry breaking and non-tachyonic scalar masses implies that $\gamma$ must be in a narrow region:

$$
0.5 \lesssim \gamma \lesssim 1.7
$$

In the above range of $\gamma$ the main properties of the toy model described in the previous section remain, while Re $z$ and its F-auxiliary component acquire non vanishing VEVs. All MSSM soft scalar masses are then equal to a universal value $m_{0}$ of the order of the gravitino mass, while the $B_{0}$ Higgs mixing parameter is also of the same order:

$$
\begin{aligned}
& m_{0}^{2}=m_{3 / 2}^{2}\left[\left(\sigma_{s}+1\right)+\frac{(\gamma+t+\gamma t)^{2}}{(1+\gamma t)^{2}}\right], \\
& A_{0}=m_{3 / 2}\left[\left(\sigma_{s}+3\right)+t \frac{\left(\gamma+t+\gamma t^{2}\right)}{1+\gamma t}\right], \\
& B_{0}=m_{3 / 2}\left[\left(\sigma_{s}+2\right)+t \frac{\left(\gamma+t+\gamma t^{2}\right)}{(1+\gamma t)}\right],
\end{aligned}
$$

where $\sigma_{s}=-3+(\rho+1)^{2}$ with $\rho=-b(s+\bar{s})$ and $t \equiv\langle\operatorname{Re} z\rangle$ determined by the minimization conditions as functions of $\gamma$. Also, $A_{0}$ is the soft trilinear scalar coupling in the standard notation, satisfying the relation [21]

$$
A_{0}=B_{0}+m_{3 / 2} \text {. }
$$

On the other hand, the gaugino masses appear to vanish at tree-level since the gauge kinetic functions are constants (see (10)). However, as mentioned in Section 3, this model is classically equivalent to the theory ${ }^{5}$

$$
\begin{aligned}
\mathcal{K} & =-\kappa^{-2} \log (s+\bar{s})+z \bar{z}+\sum_{\varphi} \varphi \bar{\varphi}, \\
W & =\left(\kappa^{-3} a(1+z)+W_{\mathrm{MSSM}}(\varphi)\right) e^{b s},
\end{aligned}
$$

obtained by applying a Kähler transformation (1) with $J=-\kappa^{-2} b s$. All classical results remain the same, such as the expressions for the scalar potential and the soft scalar masses (22), but now the shift symmetry (8) of $s$ became a gauged R-symmetry since the superpotential transforms as $W \longrightarrow W e^{-i b c \theta}$. Therefore, all fermions (including the gauginos and the gravitino) transform ${ }^{6}$ as well

\footnotetext{
${ }^{5}$ This statement is only true for supergravity theories with a non-vanishing superpotential where everything can be defined in terms of a gauge invariant function $G=\kappa^{2} \mathcal{K}+\log \left(\kappa^{6} W \bar{W}\right)[22]$.

${ }^{6}$ The chiral fermions, the gauginos and the gravitino carry a charge $b c / 2,-b c / 2$ and $-b c / 2$ respectively.
} 
under this $U(1)_{R}$, leading to cubic $U(1)_{R}^{3}$ and mixed $U(1) \times G_{\mathrm{MSSM}}$ anomalies. These anomalies are cancelled by a Green-Schwarz (GS) counter term that arises from a quantum correction to the gauge kinetic functions:

$$
f_{A}(s)=1 / g_{A}^{2}+\beta_{A} s \quad \text { with } \quad \beta_{A}=\frac{b}{8 \pi^{2}}\left(T_{R_{A}}-T_{G_{A}}\right),
$$

where $T_{G}$ is the Dynkin index of the adjoint representation, normalized to $N$ for $S U(N)$, and $T_{R}$ is the Dynkin index associated with the representation $R$ of dimension $d_{R}$, equal to $1 / 2$ for the $S U(N)$ fundamental. An implicit sum over all matter representations is understood. It follows that gaugino masses are non-vanishing in this representation, creating a puzzle on the quantum equivalence of the two classically equivalent representations. The answer to this puzzle is based on the fact that gaugino masses are present in both representations and are generated at one-loop level by an effect called Anomaly Mediation [7, 8]. Indeed, it has been argued that gaugino masses receive a one-loop contribution due to the super-Weyl-Kähler and sigma-model anomalies, given by [8]:

$$
M_{1 / 2}=-\frac{g^{2}}{16 \pi^{2}}\left[\left(3 T_{G}-T_{R}\right) m_{3 / 2}+\left(T_{G}-T_{R}\right) \mathcal{K}_{\alpha} F^{\alpha}+2 \frac{T_{R}}{d_{R}}\left(\left.\log \operatorname{det} \mathcal{K}\right|_{R}{ }^{\prime \prime}\right)_{, \alpha} F^{\alpha}\right] .
$$

The expectation value of the auxiliary field $F^{\alpha}$, evaluated in the Einstein frame is given by

$$
F^{\alpha}=-e^{\kappa^{2} \mathcal{K} / 2} g^{\alpha \bar{\beta}} \bar{\nabla}_{\bar{\beta}} \bar{W}
$$

Clearly, for the Kähler potential (10) or (24) the last term in eq. (26) vanishes. However, the second term survives due to the presence of Planck scale VEVs for the hidden sector fields $s$ and $z$. Since the Kähler potential between the two representations differs by a linear term $b(s+\bar{s})$, the contribution of the second term in eq. (26) differs by a factor

$$
\delta m_{A}=\frac{g_{A}^{2}}{16 \pi^{2}}\left(T_{G}-T_{R}\right) b e^{\kappa^{2} \mathcal{K} / 2} g^{\alpha \bar{\beta}} \bar{\nabla}_{\bar{\beta}} \bar{W}
$$

which exactly coincides with the 'direct' contribution to the gaugino masses due to the field dependent gauge kinetic function (25) (taking into account a rescaling proportional to $g_{A}^{2}$ due to the non-canonical kinetic terms).

We conclude that even though the models (10) and (24) differ by a (classical) Kähler transformation, they generate the same gaugino masses at one-loop. While the one-loop gaugino masses for the model (10) are generated entirely by eq. (26), the gaugino masses for the model (24) after a Kähler transformation have a contribution from eq. (26) as well as from a field dependent gauge kinetic term whose presence is necessary to cancel the mixed $U(1)_{R} \times G$ anomalies due to the fact that the extra $U(1)$ has become an R-symmetry giving an R-charge to all fermions in the theory. Using (26), one finds:

$$
M_{1 / 2}=-\frac{g^{2}}{16 \pi^{2}} m_{3 / 2}\left[\left(3 T_{G}-T_{R}\right)-\left(T_{G}-T_{R}\right)\left((\rho+1)^{2}+t \frac{\gamma+t+\gamma t^{2}}{1+\gamma t}\right)\right] .
$$

For $U(1)_{Y}$ we have $T_{G}=0$ and $T_{R}=11$, for $S U(2)$ we have $T_{G}=2$ and $T_{R}=7$, and for $S U(3)$ we have $T_{G}=3$ and $T_{R}=6$, such that for the different gaugino masses this gives (in a self-explanatory notation):

$$
\begin{aligned}
& M_{1}=11 \frac{g_{Y}^{2}}{16 \pi^{2}} m_{3 / 2}\left[1-(\rho+1)^{2}-\frac{t(\gamma+t+\gamma t)}{1+\gamma t}\right], \\
& M_{2}=\frac{g_{2}^{2}}{16 \pi^{2}} m_{3 / 2}\left[1-5(\rho+1)^{2}-5 \frac{t\left(\gamma+t+\gamma t^{2}\right)}{1+\gamma t}\right], \\
& M_{3}=-3 \frac{g_{3}^{2}}{16 \pi^{2}} m_{3 / 2}\left[1+(\rho+1)^{2}+\frac{t\left(\gamma+t+\gamma t^{2}\right)}{1+\gamma t}\right] .
\end{aligned}
$$




\section{Phenomenology}

The results for the soft terms calculated in the previous section, evaluated for different values of the parameter $\gamma$ are summarised in Table 1. For every $\gamma$, the corresponding $t$ and $\rho$ are calculated by imposing a vanishing cosmological constant at the minimum of the potential. The scalar soft masses and trilinear terms are then evaluated by eqs. (22) and the gaugino masses by eqs. (30). Note that the relation (23) is valid for all $\gamma$. We therefore do not list the parameter $B_{0}$.

Table 1. The soft terms (in terms of $m_{3 / 2}$ ) for various values of $\gamma$. If a solution to the RGE exists, the value of $\tan \beta$ is shown in the last columns for $\mu>0$ and $\mu<0$.

\begin{tabular}{|l|ll|lllll|rr|}
\hline$\gamma$ & $\mathrm{t}$ & $\rho$ & $m_{0}$ & $A_{0}$ & $M_{1}$ & $M_{2}$ & $M_{3}$ & $\begin{array}{r}\tan \beta \\
(\mu>0)\end{array}$ & $\begin{array}{r}\tan \beta \\
(\mu<0)\end{array}$ \\
\hline 0.6 & 0.446 & 0.175 & 0.475 & 1.791 & 0.017 & 0.026 & 0.027 & & \\
1 & 0.409 & 0.134 & 0.719 & 1.719 & 0.015 & 0.025 & 0.026 & & \\
1.1 & 0.386 & 0.120 & 0.772 & 1.701 & 0.015 & 0.024 & 0.026 & 46 & 29 \\
1.4 & 0.390 & 0.068 & 0.905 & 1.646 & 0.014 & 0.023 & 0.026 & 40 & 23 \\
1.7 & 0.414 & 0.002 & 0.998 & 1.588 & 0.013 & 0.022 & 0.025 & 36 & 19 \\
\hline
\end{tabular}

In most phenomenological studies, $B_{0}$ is substituted for $\tan \beta$, the ratio between the two Higgs VEVs, as an input parameter for the renormalization group equations (RGE) that determine the low energy spectrum of the theory. Since $B_{0}$ is not a free parameter in our theory, but is fixed by eq. (23), this corresponds to a definite value of $\tan \beta$. For more details see [23] (and references therein). The corresponding $\tan \beta$ for a few particular choices for $\gamma$ are listed in the last two columns of table 1 for $\mu>0$ and $\mu<0$ respectively. No solutions were found for $\gamma \lesssim 1.1$, for both signs of $\mu$. The lighest supersymmetric particle (LSP) is given by the lightest neutralino and since $M_{1}<M_{2}$ (see table 1) the lightest neutralino is mostly Bino-like, in contrast with a typical mAMSB (minimal anomaly mediation supersymmetry breaking) scenario, where the lightest neutralino is mostly Wino-like [24].

To get a lower bound on the stop mass, the sparticle spectrum is plotted in Figure 2 as a function of the gravitino mass for $\gamma=1.1$ and $\mu>0$ (for $\mu<0$ the bound is higher). The experimental limit on the gluino mass forces $m_{3 / 2} \gtrsim 15 \mathrm{TeV}$. In this limit the stop mass can be as low as $2 \mathrm{TeV}$. To conclude, the lower end mass spectrum consists of (very) light charginos (with a lightest chargino between 250 and $800 \mathrm{GeV})$ and neutralinos, with a mostly Bino-like neutralino as LSP $(80-230 \mathrm{GeV})$, which would distinguish this model from the mAMSB where the LSP is mostly Wino-like. These upper limits on the LSP and the lightest chargino imply that this model could in principle be excluded in the next LHC run. In order for the gluino to escape experimental bounds, the lower limit on the gravitino mass is about $15 \mathrm{TeV}$. The gluino mass is then between 1-3 TeV. This however forces the squark masses to be very high $(10-35 \mathrm{TeV})$, with the exception of the stop mass which can be relatively light $(2-15$ $\mathrm{TeV})$.

\section{Non-canonical Kähler potential for the visible sector}

As mentioned already in Section 4, an alternative way to avoid tachyonic soft scalar masses for the MSSM fields in the model (17), instead of adding the extra Palonyi-type field $z$ in the hidden sector, 


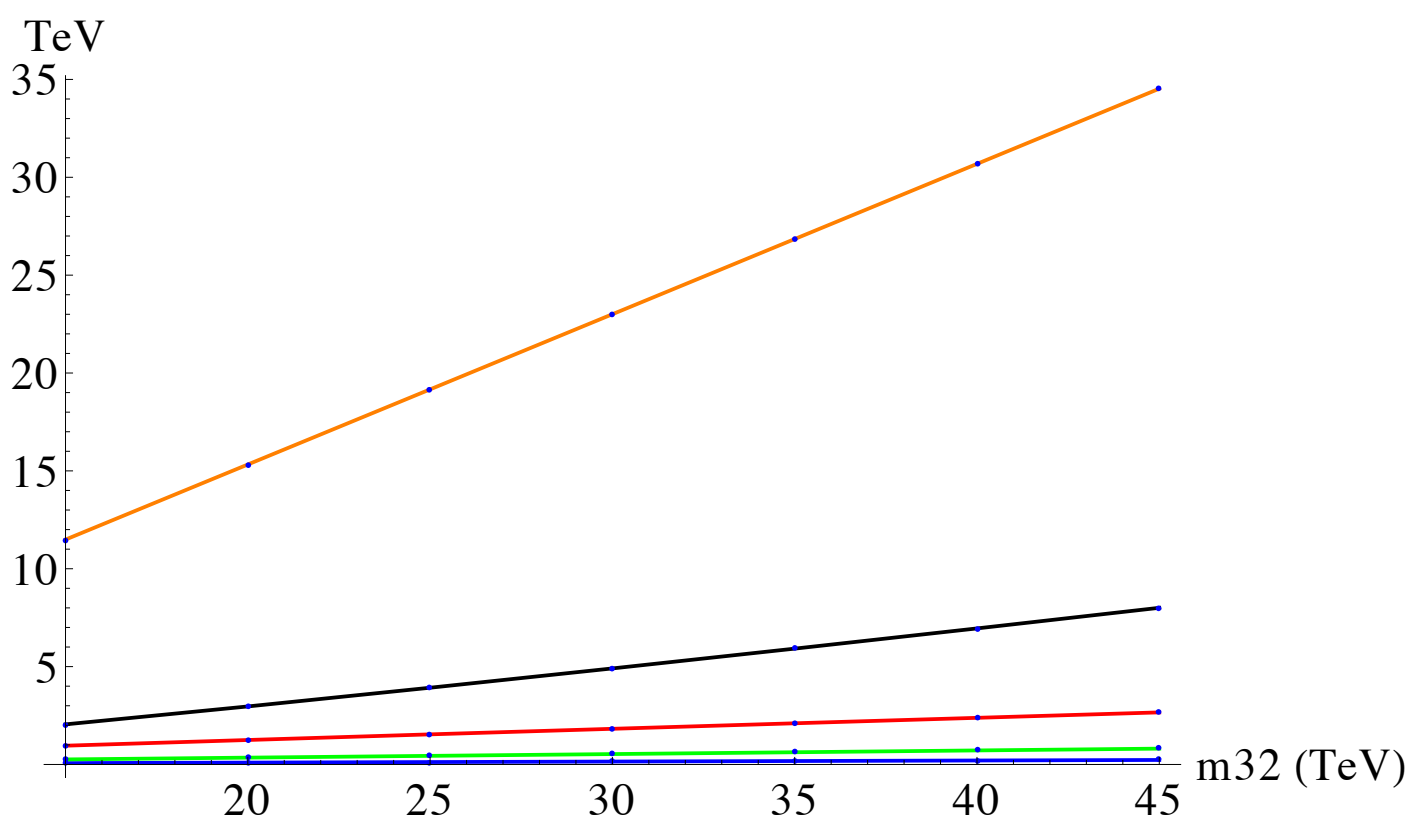

Figure 2. The masses of the sbottom (yellow), stop (black), gluino (red), lightest chargino (green) and lightest neutralino (blue) as a function of $m_{3 / 2}$ for $\gamma=1.1$ and for $\mu>0$. No solutions to the RGE were found when $m_{3 / 2} \gtrsim 45 \mathrm{TeV}$. The lower bound corresponds to a gluino mass of $1 \mathrm{TeV}$.

is by introducing non-canonical kinetic terms for the MSSM fields, such as:

$$
\begin{aligned}
\mathcal{K} & =-\kappa^{-2} \log (s+\bar{s})+\kappa^{-2} b(s+\bar{s})+(s+\bar{s})^{-v} \sum \varphi \bar{\varphi}, \\
W & =\kappa^{-3} a+W_{M S S M}, \\
f(s) & =1, \quad f_{A}(s)=1 / g_{A}^{2},
\end{aligned}
$$

where $v$ is an additional parameter of the theory, with $v=1$ corresponding to the leading term in the Taylor expansion of $-\log (s+\bar{s}-\varphi \bar{\varphi})$. Since the visible sector fields appear only in the combination $\varphi \bar{\varphi}$, their VEVs vanish provided that the scalar soft masses squared are positive. Moreover, for vanishing visible sector VEVs, the scalar potential and is minimization remains the same as in eqs. (refbsalpha). Therefore, the non-canonical Kähler potential does not change the fact that the F-term contribution to the soft scalar masses squared is negative. On the other hand, the visible fields enter in the D-term scalar potential through the derivative of the Kähler potential with respect to $s$. Even though this has no effect on the ground state of the potential, the $\varphi$-dependence of the D-term scalar potential does result in an extra contribution to the scalar masses squared which become positive

$$
v>-\frac{e^{\alpha}\left(\sigma_{s}+1\right) \alpha}{A(\alpha)(1-\alpha)} \approx 2.6
$$


The soft MSSM scalar masses and trilinear couplings in this model are:

$$
\begin{aligned}
& m_{0}^{2}=\kappa^{2} a^{2}\left(\frac{b}{\alpha}\right)\left(e^{\alpha}\left(\sigma_{s}+1\right)+v \frac{A(\alpha)}{\alpha}(1-\alpha)\right) \\
& A_{0}=m_{3 / 2}(s+\bar{s})^{v / 2}\left(\sigma_{s}+3\right) \\
& B_{0}=m_{3 / 2}(s+\bar{s})^{v / 2}\left(\sigma_{s}+2\right)
\end{aligned}
$$

where $\sigma_{s}$ is defined as in (22), eq. (20) has been used to relate the constants $a$ and $c$, and corrections due to a small cosmological constant have been neglected. A field redefinition due to a non-canonical kinetic term $g_{\varphi \bar{\varphi}}=(s+\bar{s})^{-v}$ is also taken into account. The main phenomenological properties of this model are not expected to be different from the one we analyzed in section 5 with the parameter $v$ replacing $\gamma$. Gaugino masses are still generated at one-loop level while mSUGRA applies to the soft scalar sector. We therefore do not repeat the phenomenological analysis for this model.

\section{Identifying the dilaton with the inflaton}

In the following, we study the possibility to identify the dilaton with the inflaton. We will show first that the above model does not allow slow roll inflation.

Indeed, the kinetic terms in the model (9-10) for the scalar $\phi \equiv s+\bar{s}=1 / l$ are given by

$$
\mathcal{L}_{s} / e=-g_{s \bar{s}} \partial_{\mu} s \partial^{\mu} \bar{s}=-\frac{p \kappa^{-2}}{4} \frac{1}{\phi^{2}} \partial_{\mu} \phi \partial^{\mu} \phi
$$

The canonically normalised field $\chi$ therefore satisfies $\chi=\kappa^{-1} \sqrt{\frac{p}{2}} \log \phi$, where we re-introduce the gravitational coupling $\kappa$.

The slow roll parameters are given by

$$
\begin{aligned}
& \epsilon=\frac{1}{2 \kappa^{2}}\left(\frac{d V / d \chi}{V}\right)^{2}=\frac{1}{2 \kappa^{2}}\left[\frac{1}{V} \frac{d V}{d \phi}\left(\frac{d \chi}{d \phi}\right)^{-1}\right]^{2}, \\
& \eta=\frac{1}{\kappa^{2}} \frac{V^{\prime \prime}(\chi)}{V}=\frac{1}{\kappa^{2}} \frac{1}{V}\left[\frac{d^{2} V}{d \phi^{2}}\left(\frac{d \chi}{d \phi}\right)^{-2}-\frac{d V}{d \phi} \frac{d^{2} \chi}{d \phi^{2}}\left(\frac{d \chi}{d \phi}\right)^{-3}\right],
\end{aligned}
$$

It can be shown that, when the conditions (11) and (12) are satisfied, the slow roll parameters and the potential depend only on $\rho=-b \phi$; indeed

$$
\frac{\kappa^{4} V(\rho)}{b^{3} c^{2}}=\frac{e^{-\rho}\left(A_{2}(\alpha) \rho\left(\rho^{2}+4 \rho-2\right)-2 e^{\rho}(\rho+2)^{2}\right)}{2 \rho^{3}},
$$

where $A_{2}(\alpha) \approx-50.66$ as in eq. (12). In Fig. 3, a plot is shown of $\frac{\kappa^{4} V(\rho)}{|b|^{3} c^{2}}$ as a function of $\rho$. The minimum of the potential is at $\rho_{\min } \approx 0.1832$ (see eq. (11)), while the potential has a local maximum at $\rho_{\max } \approx 0.4551$. A plot of the slow roll parameter $\eta$ (also in Fig. 3) shows that $|\eta| \ll 1$ is not satisfied. This result holds for any parameters $a, b, c$ satisfying eqs. (11) and (12). A similar analysis to the one above can be performed for $p=1$, showing that the slow roll condition $\eta \ll 1$ can not be satisfied. 


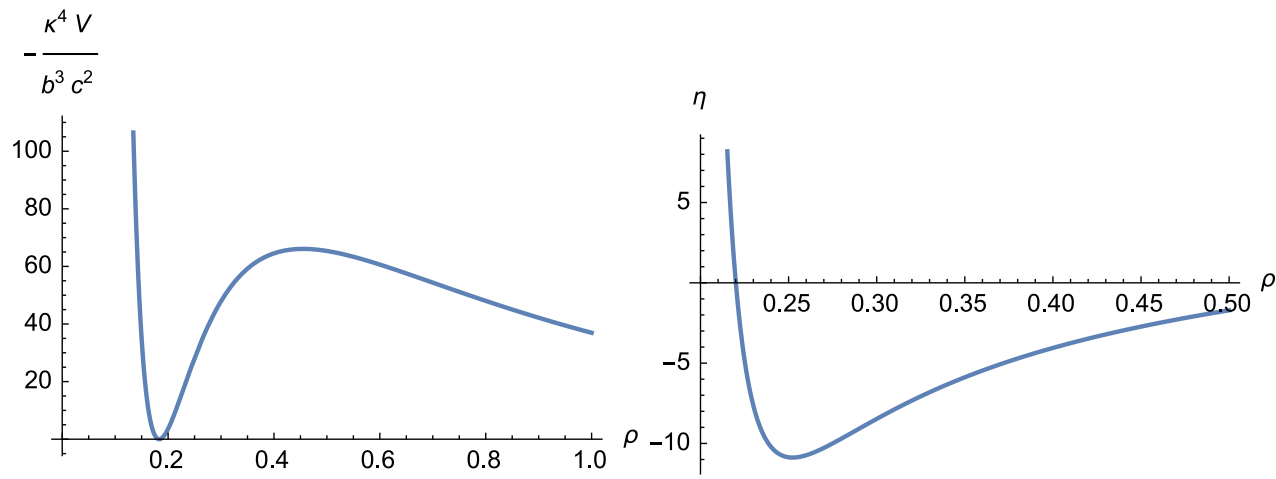

Figure 3. A plot of $-\frac{k^{4} V(\rho)}{b^{3} c^{2}}$ as a function of $\rho=-b \phi$ (left), and a plot of the slow roll parameter $\eta$ as a function of $\rho$ (right). The slow roll condition $|\eta| \ll 1$ is not satisfied for any value of the parameters $a, b, c$.

\section{Extensions of the model that satisfy the slow roll conditions}

In the previous section we showed that the slow roll conditions can not be satisfied in the minimal versions of the model. In this section we modify the above model by modifying the Kähler potential. While the superpotential is uniquely fixed (up to a Kähler transformation), the Kähler potential admits corrections that can always be put in the form

$$
\mathcal{K}=-p \kappa^{-2} \log \left(s+\bar{s}+\frac{\xi}{b} F(s+\bar{s})\right)+\kappa^{-2} b(s+\bar{s}),
$$

while the superpotential, the gauge kinetic function and moment map are given by

$$
\begin{aligned}
W & =\kappa^{-3} a, \\
f(s) & =\delta+\beta s, \\
\mathcal{P} & =\kappa^{-2} c\left(b-p \frac{1+\frac{\xi}{b} F_{s}}{s+\bar{s}+\frac{\xi}{b} F}\right),
\end{aligned}
$$

where $\mathcal{P}$ is the $U(1)$ moment map (7) and $F_{s}=\partial_{s} F(s+\bar{s})$. The scalar potential is given by $(\phi=s+\bar{s})$

$$
\begin{aligned}
V & =V_{F}+V_{D} \\
V_{F} & =\kappa^{-4} \frac{|a|^{2} e^{b \phi}}{\left(\phi+\frac{\xi}{b} F\right)^{p}}\left[-3-\frac{1}{p} \frac{\left(b(b \phi+\xi F)-p\left(b+\xi F_{\phi}\right)\right)^{2}}{\xi F_{\phi \phi}(b \phi+\xi F)-\left(b+\xi F_{\phi}\right)^{2}}\right] \\
V_{D} & =\kappa^{-4} \frac{b^{2} c^{2}}{2 \delta+\beta \phi}\left[1-p \frac{1+\frac{\xi}{b} F_{\phi}}{b \phi+\xi F}\right]^{2} .
\end{aligned}
$$

As was discussed above, we take $\delta=1, \beta=0$ for $p=1$ and $\delta=0, \beta=1$ for $p=2$.

Identifying $\operatorname{Re}(s)$ with the inverse string coupling, the function $F$ may contain perturbative contributions that can be expressed as power series of $1 /(s+\bar{s})$, as well as non-perturbative corrections which are exponentially suppressed in the weak coupling limit. The later can be either of the form $e^{-\lambda(s+\bar{s})}$ for $\lambda>0$ in the case of D-brane instantons, or of the form $e^{-\lambda(s+\bar{s})^{2}}$ in the case of (Neveu-Schwarz) NS5-brane instantons (since the closed string coupling is the square of the open string coupling). We 
have considered a generic contribution of these three different types of corrections and we found that only the last type of contributions can lead to an inflationary plateau providing sufficient inflation. The other corrections can be present but do not modify the main properties of the model (as long as weak coupling description holds). In the following section, we analyse in detailed a function $F$ describing a generic NS5-brane instanton correction to the Kähler potential.

\section{Slow-roll Inflation}

\section{$9.1 p=2$ case}

We now consider the case with

$$
F(\phi)=\exp \left(\alpha b^{2} \phi^{2}\right)
$$

where $b<0$ and $\alpha<0 . F(\phi)$ vanishes asymptotically at large $\phi$. In this case, we obtain

$$
V_{D}=\frac{\kappa^{-4} b^{3} c^{2}}{b \phi}\left[\frac{b \phi-2+\xi e^{\alpha b^{2} \phi^{2}}(1-4 \alpha b \phi)}{b \phi+\xi e^{\alpha b^{2} \phi^{2}}}\right]^{2}
$$

and

$$
V_{F}=-\frac{\kappa^{-4}|a|^{2} b^{2} e^{b \phi}}{2\left(\xi e^{\alpha b^{2} \phi^{2}}+b \phi\right)^{2}}\left[\frac{\left(b \phi+\xi e^{\alpha b^{2} \phi^{2}}(1-4 \alpha b \phi)-2\right)^{2}}{2 \alpha \xi e^{\alpha b^{2} \phi^{2}}\left(2 \alpha b^{3} \phi^{3}+\xi e^{\alpha b^{2} \phi^{2}}-b \phi\right)-1}+6\right] .
$$

There are four parameters in this model namely $\alpha, \xi, b$ and $c$. The first two parameters $\alpha$ and $\xi$ control the shape of the potential. There are some regions in the parameter space of $\alpha$ and $\xi$ that the potential satisfies the slow-roll conditions i.e. $\epsilon \ll 1$ and $|\eta| \ll 1$. In order to obtain the potential with flat plateau shape which is suitable for inflation and in agreement with Planck' 15 data, we choose

$$
\alpha \simeq-4.84 \text { and } \xi \simeq 0.025
$$

Note that in the case of $\xi=0$ and $b<0$, we can find the Minkowski minimum by solving the equations $V\left(\phi_{\min }\right)=0$ and $d V\left(\phi_{\min }\right) / d \phi=0$, where $\phi_{\min }=s_{\min }+\bar{s}_{\min }$ is the value of $\phi$ at the minimum of the potential. In the case of $\xi \neq 0$, we can not solve the equations analytically and the relations (11), (12) are not valid. We can always assume that they are modified into

$$
b \phi_{\min }=-\rho(\xi, \alpha) \quad \text { and } \quad \frac{a^{2}}{b c^{2}}=-50.66 \times \lambda(\xi, \alpha, \Lambda)^{2},
$$

where $\lambda$ takes positive values and satisfies $|\lambda-1| \ll 1$. For any given value of parameters $\xi, \alpha$ and the cosmological constant $\Lambda$, one can numerically fix the value of $\rho$ and $\lambda$. By fine-tuning the cosmological constant $\Lambda$ to be very close to zero, we can numerically solve the equations $V=0$ and $d V / d \phi=0$ for the value of $\rho$ and $\lambda$ in (44) as:

$$
\begin{aligned}
& \rho \approx 0.18 \\
& \lambda \approx 1.017
\end{aligned}
$$

From eq. (44), we can see that the third parameter, $b$, controls the vacuum expectation value $\phi_{\min }$. This can be shown in Fig. 4 where we compare the scalar potential for different values of $b$. Motivated by string theory, we have the identification $\phi \sim 1 / g_{s}$. We can choose the value of the parameter $b$ such that $\phi_{\min }$ is of the order of 10 to make sure that we are in the perturbative regime in $g_{s}$. The last 


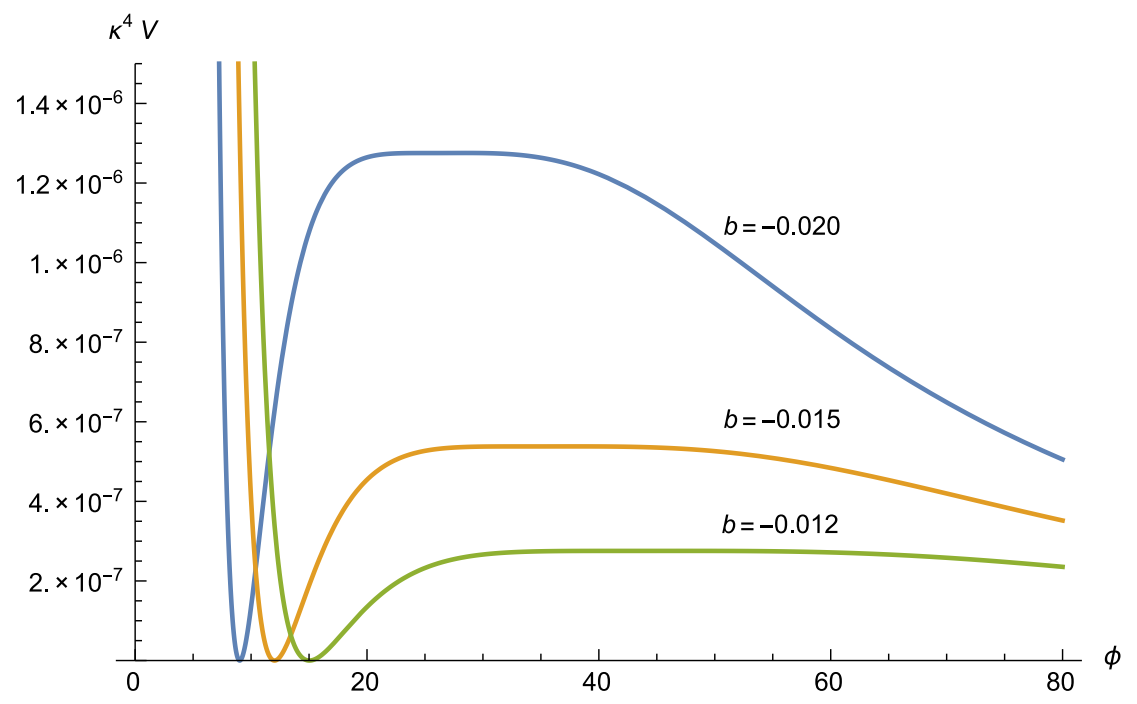

Figure 4. A plot of the scalar potential for $p=2$, with $b=-0.020, b=-0.015$ and $b=-0.012$. Note that we choose the parameters $\alpha$ and $\xi$ as in eq. (43) with $c=0.06$.

parameter, $c$, controls the overall scale of the potential but does not change its minimum and its shape. In the following, we will fix $b$ and $c$ by using the cosmological data.

In order to compare the predictions of our models with Planck' 15 data, we choose the following boundary conditions:

$$
\phi_{\text {int }}=27.32 \quad \phi_{\text {end }}=22.68
$$

The initial conditions are chosen very near the maximum on the (left) side, so that the field rolls down towards the electroweak minimum. Any initial condition on the right of the maximum may produce also inflation, but the field will roll towards the SUSY vacuum at infinity. The results are therefore very sensitive to the initial conditions (47) of the inflaton field.

The slow roll parameters are given as in equation (35). The total number of e-folds $N$ can be determined by

$$
N=\kappa^{2} \int_{\chi_{\text {end }}}^{\chi_{\text {int }}} \frac{V}{\partial_{\chi} V} d \chi=\kappa^{2} \int_{\phi_{\text {end }}}^{\phi_{\text {int }}} \frac{V}{\partial_{\phi} V}\left(\frac{d \chi}{d \phi}\right)^{2} d \phi .
$$

Note that we choose $\left|\eta\left(\chi_{\text {end }}\right)\right|=1$. We can compare the theoretical predictions of our model to the experimental results via the power spectrum of scalar perturbations of the CMB, namely the amplitude $A_{s}$ and tilt $n_{s}$, and the relative strength of tensor perturbations, i.e. the tensor-to-scalar ratio $r$. In terms of slow roll parameters, these are given by

$$
\begin{aligned}
A_{s} & =\frac{\kappa^{4} V_{*}}{24 \pi^{2} \epsilon_{*}}, \\
n_{s} & =1+2 \eta_{*}-6 \epsilon_{*}, \\
r & =16 \epsilon_{*},
\end{aligned}
$$


Table 2. The theoretical predictions for $p=2$, with $b=-0.0182, c=0.61 \times 10^{-13}$, and $\alpha$, $\xi$ given in eq. (43).

\begin{tabular}{|c|c|c|}
\hline$n_{s}$ & $r$ & $A_{s}$ \\
\hline 0.965 & $2.969 \times 10^{-23}$ & $2.259 \times 10^{-9}$ \\
\hline
\end{tabular}

where all parameters are evaluated at the field value $\chi_{\text {int }}$.

In order to satisfy Planck' 15 data, we choose the parameters $b=-0.0182, c=0.61 \times 10^{-13}$. The value of the slow-roll parameters at the beginning of inflation are

$$
\epsilon\left(\phi_{\text {int }}\right) \simeq 1.86 \times 10^{-24} \text { and } \eta\left(\phi_{\text {int }}\right) \simeq-1.74 \times 10^{-2} .
$$

The total number of e-folds $N$, the scalar power spectrum amplitude $A_{s}$, the spectral index of curvature perturbation $n_{s}$ and the tensor-to-scalar ratio $r$ are calculated and summarised in Table 2, in agreement with Planck' 15 data [25]. Fig. 5 shows that our predictions for $n_{s}$ and $r$ are within $1 \sigma$ C.L. of Planck ' 15 contours with the total number of e-folds $N \approx 1075$. Note that $N$ is the total number of e-folds from $\phi_{\text {int }}$ to $\phi_{\text {end }}$. However the number of e-folds associated with the CMB observation corresponds to a period between the time of horizon crossing and the end of inflation, which is much smaller than 1075. According to general formula in [25], the number of e-folds between the horizon crossing and the end of inflation is roughly estimated to be around 50-60.

We would like to remark that the parameter $c$ also controls the gravitino mass at the minimum of the potential around $O(10) \mathrm{TeV}$. Indeed, the gravitino mass is written as

$$
m_{3 / 2}=\kappa^{2} e^{\kappa^{2} \mathcal{K} / 2} W=\frac{1}{\kappa}\left(\frac{a b e^{b \phi / 2}}{b \phi+\xi F(\phi)}\right) .
$$

For $b=-0.0182$, we get $\phi_{\min } \approx 9.91134$ and the gravitino mass at the minimum of the potential

$$
\left\langle m_{3 / 2}\right\rangle \approx 14.98 \mathrm{TeV}
$$

The Hubble parameter during inflation (evaluated at $\phi_{*}=\phi_{\text {int }}$ ) is

$$
H_{*}=\kappa \sqrt{V_{*} / 3}=1.38 \mathrm{TeV}
$$

This shows that our predicted scale for inflation is of the order of TeV. The mass of gravitino during the inflation $m_{3 / 2}^{*}=4.15 \mathrm{TeV}$ is higher than the inflation scale, and the gauge boson mass is $M_{A_{\mu}}^{*}=$ $3.12 \mathrm{TeV} .{ }^{7}$ In fact, the gauge boson acquires a mass due to a Stueckelberg mechanism by eating the imaginary component of $s$, where its mass at the minimum of the potential is given by

$$
\left\langle M_{A_{\mu}}\right\rangle=15.48 \mathrm{TeV}
$$

As a result, the model essentially contains only one scalar field $\operatorname{Re}(s)$, which is the inflaton. This is in contrast with other supersymmetric models of inflation, which usually contain at least two real scalars [26]. ${ }^{8}$

\footnotetext{
${ }^{7}$ The gauge boson mass is given by $m_{A_{\mu}}=\sqrt{2 g_{s} c^{2} / \operatorname{Re}(s)}$.

${ }^{8}$ This is because a chiral multiplet contains a complex scalar.
} 


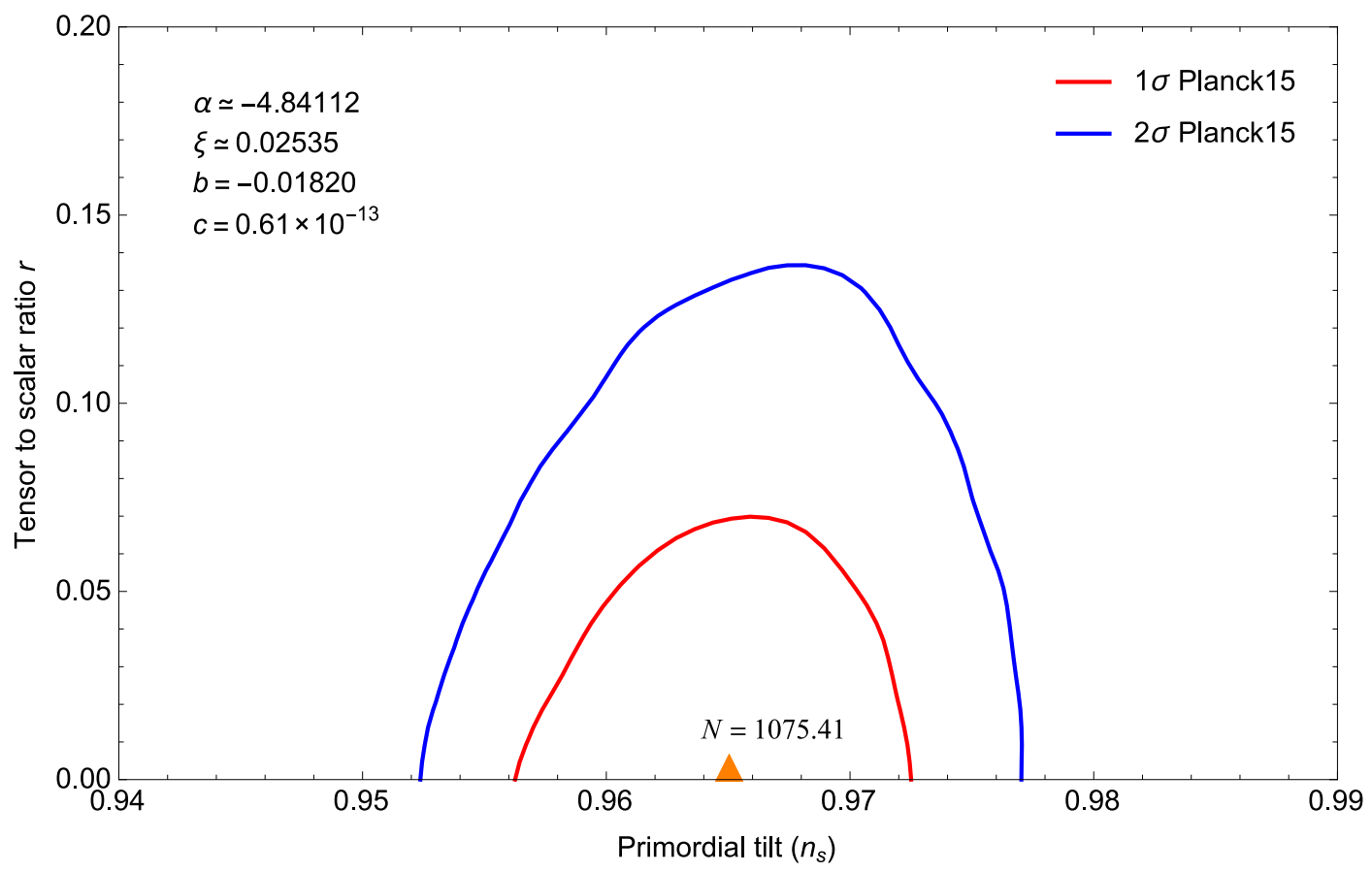

Figure 5. We plot the theoretical predictions for the case $p=2$, shown in Table 2, in the $n_{s}-r$ plane together with the Planck' 15 results for TT, TE, EE, + lowP and assuming $\Lambda \mathrm{CDM}+\mathrm{r}$.

\section{$9.2 \mathrm{p}=1$ case}

In this case, we obtain

$$
V_{D}=\frac{\kappa^{-4} b^{2} c^{2}}{2}\left[\frac{b \phi-1+\xi e^{\alpha b^{2} \phi^{2}}(1-2 \alpha b \phi)}{b \phi+\xi e^{\alpha b^{2} \phi^{2}}}\right]^{2}
$$

and

$$
V_{F}=-\frac{\kappa^{-4}|a|^{2} b e^{b \phi}}{\xi e^{\alpha b^{2} \phi^{2}}+b \phi}\left[\frac{\left(b \phi+\xi e^{\alpha b^{2} \phi^{2}}(1-2 \alpha b \phi)-1\right)^{2}}{2 \alpha \xi e^{\alpha b^{2} \phi^{2}}\left(2 \alpha b^{3} \phi^{3}+\xi e^{\alpha b^{2} \phi^{2}}-b \phi\right)-1}+3\right] .
$$

The potential has similar properties with the $p=2$ case although it may give different phenomenological results at low energy. Similar to the previous case, the relations (20) are not valid when $\xi \neq 0$ and we assume that they are modified into

$$
b \phi_{\min }=-\rho(\xi, \alpha) \text { and } \frac{b c^{2}}{a^{2}} \simeq-0.359 \times \lambda(\xi, \alpha, \Lambda)^{-2} .
$$

By choosing $\alpha=-0.781$ and $\xi=0.3023$ and tuning the cosmological constant $\Lambda$ to be very close to zero, we can numerically fix $\rho \approx 0.56$ and $\lambda \approx 1.29$ for this case. The gravitino mass for $p=1$ case can be written as

$$
m_{3 / 2}=\kappa^{2} e^{\kappa^{2} \mathcal{K} / 2} W=\frac{1}{\kappa}\left(\frac{a \sqrt{b} e^{b \phi / 2}}{\sqrt{b \phi+\xi F(\phi)}}\right) .
$$


Table 3. The theoretical predictions for $p=1$ with $b=-0.0234, c=1 \times 10^{-13}, \alpha=-0.781$ and $\xi=0.3023$.

\begin{tabular}{|c|c|c|}
\hline$n_{s}$ & $r$ & $A_{s}$ \\
\hline 0.959 & $4.143 \times 10^{-22}$ & $2.205 \times 10^{-9}$ \\
\hline
\end{tabular}

By choosing the parameters $b=-0.0234, c=1 \times 10^{-13}$, the gravitino mass at the minimum of the potential is

$$
\left\langle m_{3 / 2}\right\rangle=18.36 \mathrm{TeV}
$$

with $\phi_{\min } \approx 21.53$, and

$$
\left\langle M_{A_{\mu}}\right\rangle=36.18 \mathrm{TeV} .
$$

By choosing appropriate boundary conditions, we find

$$
\phi_{\text {int }}=64.53 \text { and } \phi_{\text {end }}=50.99
$$

As summarised in Table 3, the predictions for the $p=1$ case are similar to those of $p=2$, in agreement with Planck' 15 data with the total number of e-folds $N \approx 888$. In this case, the Hubble parameter during inflation is

$$
H_{*}=\kappa \sqrt{V_{*} / 3}=5.09 \mathrm{TeV} .
$$

Note that for the $p=1$ case, the mass of the gauge boson is $M_{A_{\mu}}^{*}=6.78 \mathrm{TeV}$, and the mass of the gravitino during inflation is $m_{3 / 2}^{*}=4.72 \mathrm{TeV}$.

\subsection{SUGRA spectrum}

The above model can be coupled to MSSM, as described in section 4:

$$
\begin{aligned}
\mathcal{K} & =\mathcal{K}(s+\bar{s})+\sum \varphi \bar{\varphi}, \\
W & =W_{h}(s)+W_{\text {MSSM }} .
\end{aligned}
$$

The soft supersymmetry breaking terms can then be calculated as follows

$$
\begin{aligned}
m_{0}^{2} & =e^{\kappa^{2} \mathcal{K}}\left(-2 \kappa^{4} W_{h}(s) \bar{W}_{h}(s)+\kappa^{2} g^{s \bar{s}}\left|\nabla_{s} W_{h}\right|^{2}\right), \\
A_{0} & =\kappa^{2} e^{\kappa^{2} \mathcal{K} / 2} g^{s \bar{s}} K_{s}\left(\bar{W}_{\bar{s}}+\kappa^{2} K_{s} \bar{W}\right), \\
B_{0} & =\kappa^{2} e^{\kappa^{2} \mathcal{K} / 2}\left(g^{s \bar{s}} K_{s}\left(\bar{W}_{\bar{s}}+\kappa^{2} K_{s} \bar{W}\right)-\bar{W}\right) .
\end{aligned}
$$

For $p=2$ the Lagrangian contains a Green-Schwarz term eq. (18), and the theory is not gauge invariant (without the inclusion of extra fields that are charged under the $U(1)$ ). We therefore focus on $p=1$. The soft terms can be written in terms of the gravitino mass (see eq. (53))

$$
\begin{aligned}
m_{0}^{2} & =m_{3 / 2}^{2}[-2+C], \\
A_{0} & =m_{3 / 2} C, \\
B_{0} & =A_{0}-m_{3 / 2},
\end{aligned}
$$

where

$$
C=-\left.\frac{\left(-\xi e^{\alpha b^{2} \phi^{2}}+b \phi\left(4 \alpha \xi e^{\alpha b^{2} \phi^{2}}-1\right)+2\right)^{2}}{4 \alpha \xi^{2} e^{2 \alpha b^{2} \phi^{2}}-4 \alpha b \xi \phi e^{\alpha b^{2} \phi^{2}}+8 \alpha^{2} b^{3} \xi \phi^{3} e^{\alpha b^{2} \phi^{2}}-2}\right|_{\phi=\phi_{\min }}
$$


Using the parameters presented in section 9.2, we find $m_{3 / 2}=18.36 \mathrm{TeV}$ and $C=1.53$. For $\xi=0$ the model reduces to the one analysed in section 4, where one has $C=1.52$ and $m_{3 / 2}=17.27 \mathrm{TeV}$ (with $\phi_{\min }=9.96$ ). Moreover, the scalar soft mass is tachyonic. This can be solved either by introducing an extra Polonyi-like field, or by allowing a non-canonical Kähler potential for the MSSM-like fields $\varphi$. The resulting low energy spectrum is expected to be similar to the one described in sections 4 and 5 . We do not perform this analysis, but only summarise the results.

Since the tree-level contribution to the gaugino masses vanishes, their mass is generated at oneloop by the so-called 'Anomaly Mediation' contribution (26). As a result, the spectrum consists of very light neutralinos $\left(O\left(10^{2}\right) \mathrm{GeV}\right)$, of which the lightest (a mostly Bino-like neutralino) is the LSP dark matter candidate, slightly heavier charginos and a gluino in the $1-3 \mathrm{TeV}$ range. The squarks are of the order of the gravitino mass $(\sim 10 \mathrm{TeV})$, with the exception of the stop squark which can be as light as $2 \mathrm{TeV}$.

\section{Symmetric versus non-symmetric point}

Here, we generalise the above model of inflation and we are interested in supergravity theories containing a single chiral multiplet transforming under a gauged R-symmetry with a corresponding abelian vector multiplet [16]. We assume that the chiral multiplet $\mathcal{X}$ (with scalar component $X$ ) transforms as:

$$
X \longrightarrow X e^{-i q \omega}
$$

where $q$ is its charge, and $\omega$ is the gauge parameter.

The Kähler potential is therefore a function of $X \bar{X}$, while the superpotential is constrained to be of the form $X^{b}$ :

$$
\begin{aligned}
& \mathcal{K}=\mathcal{K}(X \bar{X}), \\
& W=\kappa^{-3} f X^{b},
\end{aligned}
$$

where $X$ is a dimensionless field and $\kappa^{-1}=m_{p}=2.4 \times 10^{15} \mathrm{TeV}$ is the (reduced) Planck mass. For $b \neq 0$, the gauge symmetry eq. (69) becomes a gauged R-symmetry. The gauge kinetic function can have a constant contribution as well as a contribution proportional to $\ln X$

$$
f(X)=\gamma+\beta \ln X .
$$

The latter contribution proportional to $\beta$ is not gauge invariant and can be used as a Green-Schwarz counter term to cancel possible anomalies. One can show however that the constant $\beta$ is fixed to be very small by anomaly cancellation conditions and does not change our results [16]. We will therefore omit this term in our analysis below.

We are interested in the general properties of supergravity theories of inflation that are of the above form. Before performing our analysis, a distinction should be made concerning the initial point where slow-roll inflation starts. The inflaton field (which will turn out to be $\rho$, where $X=\rho e^{i \theta}$ ) can either have its initial value close to the symmetric point where $X=0$, or at a generic point $X \neq 0$. The minimum of the potential, however, is always at a nonzero point $X \neq 0$. This is because at $X=0$ the negative contribution to the scalar potential vanishes and no cancellation between F-term and D-term is possible. The supersymmetry breaking scale is therefore related to the cosmological constant as $\kappa^{-2} m_{3 / 2}^{2} \approx \Lambda$. One could in principle assume that the value of the potential at its minimum is of the order of the supersymmetry breaking scale. However, in this case additional corrections are needed 
to bring down the minimum of the potential to the present value of the cosmological constant, and we therefore do not discuss this possibility.

In the first case, inflation starts near $X=0$, and the inflaton field will roll towards a minimum of the potential at $X \neq 0$. On the other hand, in the second case inflation will start at a generic point $X \neq 0$. In order to make easier contact with the model discussed in the previous sections, it is convenient to work with another chiral superfield $S$, which is invariant under a shift symmetry

$$
S \longrightarrow S-i c \alpha
$$

by performing a field redefinition

$$
X=e^{S} .
$$

In this case the most general Kähler potential and superpotential are of the form

$$
\begin{aligned}
& \mathcal{K}=\mathcal{K}(S+\bar{S}), \\
& W=\kappa^{-3} a e^{b S} .
\end{aligned}
$$

Note that this field redefinition is not valid at the symmetric point $X=0$ for the first case.

\section{Case 1: Inflation near the symmetric point}

\subsection{Slow roll parameters}

In this section we derive the conditions that lead to slow-roll inflation scenarios, where the start of inflation is near a local maximum of the potential at $X=0$. Since the superpotential has charge 2 under R-symmetry, one has $\langle W\rangle=0$ as long as R-symmetry is preserved. Therefore, $\langle W\rangle$ can be regarded as the order parameter of R-symmetry breaking. On the other hand, the minimum of the potential requires $\langle W\rangle \neq 0$ and broken R-symmetry. It is therefore attractive to assume that at earlier times R-symmetry was a good symmetry, switching off dangerous corrections to the potential. As similar approach was followed in [27], where a discrete R-symmetry is assumed. Instead, we assume a gauged R-symmetry which is spontaneously broken at the minimum of the potential.

While the superpotential is uniquely fixed in eq. (70), the Kähler potential is only fixed to be of the form $\mathcal{K}(X \bar{X})$. We expand the Kähler potential as follows

$$
\begin{aligned}
\mathcal{K}(X, \bar{X}) & =\kappa^{-2} X \bar{X}+\kappa^{-2} A(X \bar{X})^{2}, \\
W(X) & =\kappa^{-3} f X^{b}, \\
f(X) & =1,
\end{aligned}
$$

where $A$ and $f$ are constants. The gauge kinetic function is taken to be constant since it was shown that the coefficient $\beta$ in front of the logarithmic term in eq. (71) is fixed to be very small by anomaly cancellation conditions [16]. As far as the scalar potential is concerned, the coefficient $\gamma$ can be absorbed in other parameters of the theory. We therefore take $\gamma=1$.

The scalar potential is given by

$$
\mathcal{V}=\mathcal{V}_{F}+\mathcal{V}_{D}
$$

where

$$
\mathcal{V}_{F}=\kappa^{-4} f^{2}(X \bar{X})^{b-1} e^{X \bar{X}(1+A X \bar{X})}\left[-3 X \bar{X}+\frac{(b+X \bar{X}(1+2 A X \bar{X}))^{2}}{1+4 A X \bar{X}}\right]
$$


and

$$
\mathcal{V}_{D}=\kappa^{-4} \frac{q^{2}}{2}[b+X \bar{X}(1+2 A X \bar{X})]^{2}
$$

The superpotential is not gauge invariant under the $U(1)$ gauge symmetry. Instead it transforms as

$$
W \rightarrow W e^{-i q b w} .
$$

Therefore, the $U(1)$ is a gauged R-symmetry which we will further denote as $U(1)_{R}$. From $W_{X} k_{R}^{X}=$ $-r_{R} \kappa^{2} W$, where $k_{R}^{X}=-i q X$ is the Killing vector for the field $X$ under the R-symmetry, $r_{R}=i \kappa^{-2} \xi_{R}$ with $\kappa^{-2} \xi_{R}$ the Fayet-Iliopoulos contribution to the scalar potential, and $W_{X}$ is short-hand for $\partial W / \partial X$, we find

$$
r_{R}=i \kappa^{-2} q b
$$

A consequence of the gauged R-symmetry is that the superpotential coupling $b$ enters the D-term contribution of the scalar potential as a constant Fayet-Iliopoulos contribution. ${ }^{9}$

Note that the scalar potential is only a function of the modulus of $X$ and that the potential contains a Fayet-Iliopoulos contribution for $b \neq 0$. Moreover, its phase will be 'eaten' by the $U(1)$ gauge boson upon a field redefinition of the gauge potential similarly to the standard Higgs mechanism. After performing a change of field variables

$$
X=\rho e^{i \theta}, \quad \bar{X}=\rho e^{-i \theta}, \quad(\rho \geq 0)
$$

the scalar potential is a function of $\rho$,

$$
\kappa^{4} \mathcal{V}=f^{2} \rho^{2(b-1)} e^{\rho^{2}+A \rho^{4}}\left(-3 \rho^{2}+\frac{\left(b+\rho^{2}+2 A \rho^{4}\right)^{2}}{1+4 A \rho^{2}}\right)+\frac{q^{2}}{2}\left(b+\rho^{2}+2 A \rho^{4}\right)^{2}
$$

Since we assume that inflation starts near $\rho=0$, we require that the potential eq. (82) has a local maximum at this point. It turns out that the potential only allows for a local maximum at $\rho=0$ when $b=1$. For $b<1$ the potential diverges when $\rho$ goes to zero. For $1<b<1.5$ the first derivative of the potential diverges, while for $b=1.5$, one has $V^{\prime}(0)=\frac{9}{4} f^{2}+\frac{3}{2} q^{2}>0$, and for $b>1.5$, on has $V^{\prime \prime}(0)>0$. We thus take $b=1$ and the scalar potential reduces to

$$
\kappa^{4} \mathcal{V}=f^{2} e^{\rho^{2}+A \rho^{4}}\left(-3 \rho^{2}+\frac{\left(1+\rho^{2}+2 A \rho^{4}\right)^{2}}{1+4 A \rho^{2}}\right)+\frac{q^{2}}{2}\left(1+\rho^{2}+2 A \rho^{4}\right)^{2} .
$$

Note that in this case the the superpotential is linear $W=f X$, describing the sgoldstino (up to an additional low-energy constraint) [30]. Indeed, modulo a D-term contribution, the inflaton in this model is the superpartner of the goldstino. In fact, for $q=0$ the inflaton reduces to the partner of the goldstino as in Minimal Inflation models [31]. The important difference however is that this is a microscopic realisation of the identification of the inflaton with the sgoldstino, and that the so-called $\eta$-problem is avoided (see discussion below).

\footnotetext{
${ }^{9}$ For other studies of inflation involving Fayet-Iliopoulos terms see for example [28], or [29] for more recent work. Moreover, our motivations have some overlap with [27], where inflation is also assumed to start near an R-symmetric point at $X=0$. However, this work uses a discrete R-symmetry which does not lead to Fayet-Iliopoulos terms.
} 
The kinetic terms for the scalars can be written as ${ }^{10}$

$$
\mathcal{L}_{\text {kin }}=-g_{X \bar{X}} \hat{\partial}_{\mu} X \hat{\partial}^{\mu} X=-g_{X \bar{X}}\left[\partial_{\mu} \rho \partial^{\mu} \rho+\rho^{2}\left(\partial_{\mu} \theta+q A_{\mu}\right)\left(\partial^{\mu} \theta+q A^{\mu}\right)\right] .
$$

It was already anticipated above that the phase $\theta$ plays the role of the longitudinal component of the gauge field $A_{\mu}$, which acquires a mass by a Brout-Englert-Higgs mechanism.

We now interpret the field $\rho$ as the inflaton. It is important to emphasise that, in contrast with usual supersymmetric theories of inflation where one necessarily has two scalar degrees of freedom resulting in multifield inflation [26], our class of models contains only one scalar field $\rho$ as the inflaton. In order to calculate the slow-roll parameters, one needs to work with the canonically normalised field $\chi$ satisfying

$$
\frac{d \chi}{d \rho}=\sqrt{2 g_{X \bar{X}}}
$$

The slow-roll parameters are given in terms of the canonical field $\chi$ by

$$
\epsilon=\frac{1}{2 \kappa^{2}}\left(\frac{d V / d \chi}{V}\right)^{2}, \quad \eta=\frac{1}{\kappa^{2}} \frac{d^{2} V / d \chi^{2}}{V} .
$$

Since we assume inflation to start near $\rho=0$, we expand

$$
\begin{aligned}
& \epsilon=4\left(\frac{-4 A+x^{2}}{2+x^{2}}\right)^{2} \rho^{2}+O\left(\rho^{4}\right), \\
& \eta=2\left(\frac{-4 A+x^{2}}{2+x^{2}}\right)+O\left(\rho^{2}\right),
\end{aligned}
$$

where we defined $q=f x$. Notice that for $\rho \ll 1$ the $\epsilon$ parameter is very small, while the $\eta$ parameter can be made small by carefully tuning the parameter $A$. Any higher order corrections to the Kähler potential do not contribute to the leading contributions in the expansion near $\rho=0$ for $\eta$ and $\epsilon$. Such corrections can therefore be used to alter the potential near its minimum, at some point $X \neq 0$ without influencing the slow-roll parameters.

\section{A comment on the $\eta$-problem in Supergravity}

A few words are now in order concerning the $\eta$-problem [32]. The $\eta$ problem in $\mathcal{N}=1$ supergravity is often stated as follows (see for example [33]): If, for instance, a theory with a single chiral multiplet with scalar component $\varphi$ is taken, then the Kähler potential can be expanded around a reference location $\varphi=0$ as $\mathcal{K}=\mathcal{K}(0)+\mathcal{K}_{\varphi \bar{\varphi}}(0) \varphi \bar{\varphi}+\ldots$. The Lagrangian becomes

$$
\mathcal{L}=-\partial_{\mu} \phi \partial^{\mu} \bar{\phi}-\mathcal{V}(0)\left(1+\kappa^{2} \phi \bar{\phi}+\cdots\right),
$$

where $\phi$ is the canonically normalised field $\phi \bar{\phi}=\mathcal{K}_{\varphi \bar{\varphi}}(0) \varphi \bar{\varphi}$, and the ellipses stand for extra terms in the expansion coming from $\mathcal{K}$ and $W$. Following this argument, the mass $m_{\phi}$ turns out to be proportional to the Hubble scale

$$
m_{\phi}^{2}=\kappa^{2} \mathcal{V}(0)+\cdots=3 H^{2}+\ldots,
$$

\footnotetext{
${ }^{10}$ The covariant derivative is defined as $\hat{\partial}_{\mu} X=\partial_{\mu} X-A_{\mu} k_{R}^{X}$, where $k_{R}^{X}=-i q X$ is the Killing vector for the U(1) transformation eq. (69).
} 
and therefore

$$
\eta=\frac{m_{\phi}^{2}}{3 H^{2}}=1+\ldots
$$

Or otherwise stated, this leading contribution of order 1 to the $\eta$-parameter has its origin from the fact that the F-term contribution to the scalar potential contains an exponential factor $e^{\mathcal{K}}: \mathcal{V}=e^{X \bar{X}+\ldots}[\ldots]$ resulting in its second derivative $\mathcal{V}_{X \bar{X}}=V[1+\ldots]$.

However, in our model the factor ' 1 ' drops out for the particular choice $b=1$ in the superpotential ${ }^{11}$, resulting in an inflaton mass $m_{\rho}^{2}$ which is determined by the next term $A(X \bar{X})^{2}$ in the expansion of the Kähler potential,

$$
\begin{aligned}
& m_{\chi}^{2}=\left(-4 A+x^{2}\right) \kappa^{-2} f^{2}+O\left(\rho^{2}\right), \\
& H^{2}=\frac{\kappa^{-2} f^{2}}{6}\left(2+x^{2}\right)+O\left(\rho^{2}\right) .
\end{aligned}
$$

As a result, there are two ways to evade the $\eta$-problem:

- First, one can obtain a small $\eta$ by having a small $q \ll f$, while $A$ should be of order $O\left(10^{-1}\right)$. In this case, the rôle of the gauge symmetry is merely to constrain the form of the Kähler potential and the superpotential, and to provide a Higgs mechanism that eliminates the extra scalar (phase) degree of freedom.

- Alternatively there could be a cancellation between $q^{2}$ and $4 A f^{2}$.

Since $A$ is the second term in the expansion of the Kähler potential eq. (75), it is natural to be of order $O\left(10^{-1}\right)$ and therefore providing a solution to the $\eta$-problem.

Note that the mass of the inflaton given in eqs. (91) is only valid during inflation at small $\rho$. The mass of the inflaton at its VEV will be affected by additional corrections that are needed to obtain in particular a vanishing value for the scalar potential at its minimum [16].

\section{The upper bound on the tensor-to-scalar ratio}

Before moving on to the next section, let us focus on the approximation at $\rho \ll 1$ where the perturbative expansion of the slow-roll parameters in eqs. (87) is valid, and assume that the horizon exit occurs at the field value $\rho_{*}$ very close to the maximum $\rho=0$. In this approximation, eqs. (87) become

$$
\epsilon(\rho) \approx \epsilon^{\mathrm{pert}}(\rho)=\left|\eta_{*}\right|^{2} \rho^{2}, \quad \eta(\rho) \approx \eta_{*},
$$

where the asterisk refers to the value of parameters evaluated at the horizon exit.

To discuss the upper bound on the tensor-to-scalar ratio, it is convenient to divide the region $\left[\rho=0, \rho_{\text {end }}\right]$ into two regions: one is $\left[0, \rho_{\mathrm{p}}\right]$, where the approximation (92) is valid, and the other is the rest $\left[\rho_{\mathrm{p}}, \rho_{\mathrm{end}}\right]$. Here $\rho_{\text {end }}$ means the inflation end. Note that $\rho_{\mathrm{p}}<\rho_{\text {end }}$ because the approximation (92) breaks down before the end of inflation where $\epsilon\left(\rho_{\text {end }}\right)=1$ or $\left|\eta\left(\rho_{\text {end }}\right)\right|=1$. In terms of this division, the number of e-folds from the horizon exit to the end of inflation can be approximated by

$$
N_{\mathrm{CMB}} \simeq N^{\mathrm{pert}}\left(\rho_{*}, \rho_{\mathrm{p}}\right)+\kappa \int_{\chi_{\mathrm{p}}}^{\chi_{\mathrm{end}}} \frac{d \chi}{\sqrt{2 \epsilon(\chi)}},
$$

\footnotetext{
${ }^{11}$ Note that in hybrid inflation models the $\eta$-problem is also evaded by a somewhat similar way, but these models generally include several scalar fields (and superfields) besides the inflaton (see e.g. [34]).
} 
where we introduced

$$
N^{\text {pert }}\left(\rho_{1}, \rho_{2}\right)=\kappa \int_{\chi_{1}}^{\chi_{2}} \frac{d \chi}{\sqrt{2 \epsilon^{\text {pert }}(\chi)}}=\frac{1}{\left|\eta_{*}\right|} \ln \left(\frac{\rho_{2}}{\rho_{1}}\right) .
$$

Here $\chi$ is the canonically normalised field defined by eq. (85). Let us next focus on the region $\left[\rho_{\mathrm{p}}, \rho_{\mathrm{end}}\right]$. It is natural to expect the following inequality

$$
\kappa \int_{\chi_{\mathrm{p}}}^{\chi_{\mathrm{end}}} \frac{d \chi}{\sqrt{2 \epsilon(\chi)}} \lesssim \kappa \int_{\chi_{\mathrm{p}}}^{\chi_{\mathrm{end}}} \frac{d \chi}{\sqrt{2 \epsilon^{\mathrm{pert}}(\chi)}} .
$$

This is based on the following observation. The right hand side describes a hypothetical situation, as if the slow-roll condition were valid throughout the inflation until its end. But since in the actual inflation the slow-roll condition breaks down in the region $\left[\rho_{\mathrm{p}}, \rho_{\mathrm{end}}\right]$, the actual number of e-folds in this region will be smaller than that in the hypothetical situation. Adding $N^{\text {pert }}\left(\rho_{*}, \rho_{\mathrm{p}}\right)$ to the both hand sides of (95) and using (93), we find

$$
N_{\mathrm{CMB}} \lesssim \frac{1}{\left|\eta_{*}\right|} \ln \left(\frac{\rho_{\mathrm{end}}}{\rho_{*}}\right) .
$$

Using (92) and the definition of the tensor-to-scalar ratio $r=16 \epsilon_{*}$, we obtain the upper bound:

$$
r \lesssim 16\left(\left|\eta_{*}\right| \rho_{\mathrm{end}} e^{-\left|\eta_{*}\right| N_{\mathrm{CMB}}}\right)^{2}
$$

To satisfy CMB data, let us choose $\eta=-0.02$ and $N_{\mathrm{CMB}} \approx 50$. Assuming $\rho_{\text {end }} \lesssim 1 / 2$, we obtain the upper bound $r \lesssim 10^{-4}$. Note that this is a little bit lower than the Lyth bound [35] for small field inflation, $r \lesssim 10^{-3}$. From the upper bound on $r$, we can also find the upper bound on the Hubble parameter as follows. In general, the power spectrum amplitude $A_{s}$ is related to the Hubble parameter at horizon exit $H_{*}$ by

$$
A_{s}=\frac{2 \kappa^{2} H_{*}^{2}}{\pi^{2} r} .
$$

Combining this with the upper bound $r \lesssim 10^{-4}$ and the value $A_{s}=2.2 \times 10^{-9}$ by CMB data, we find the upper bound on the Hubble parameter $H_{*} \lesssim 10^{9} \mathrm{TeV}$.

In Ref. [16], we will also find the lower bound $r \gtrsim 10^{-9}$ (equivalently $H_{*} \gtrsim 10^{7} \mathrm{TeV}$ ), based on an model-independent argument. This bound can be lowered at the cost of naturalness between parameters in the potential.

\section{References}

[1] I. Antoniadis, N. Arkani-Hamed, S. Dimopoulos and G. R. Dvali, "New dimensions at a millimeter to a Fermi and superstrings at a TeV,” Phys. Lett. B 436 (1998) 257 [arXiv:hep-ph/9804398].

[2] I. Antoniadis and S. P. Patil, Eur. Phys. J. C75 (2015) 182 [arXiv:1410.8845 [hep-th]].

[3] I. Antoniadis and R. Knoops, Nucl. Phys. B 886 (2014) 43 [arXiv:1403.1534 [hep-th]].

[4] F. Catino, G. Villadoro and F. Zwirner, JHEP 1201 (2012) 002 [arXiv:1110.2174 [hep-th]], G. Villadoro and F. Zwirner, Phys. Rev. Lett. 95 (2005) 231602 [hep-th/0508167].

[5] I. Antoniadis, D. M. Ghilencea and R. Knoops, JHEP 1502 (2015) 166 [arXiv:1412.4807 [hepth]].

[6] I. Antoniadis and R. Knoops, Nucl. Phys. B 902 (2016) 69 [arXiv:1507.06924 [hep-ph]]. 
[7] L. Randall and R. Sundrum, Nucl. Phys. B 557 (1999) 79 [hep-th/9810155], G. F. Giudice, M. A. Luty, H. Murayama and R. Rattazzi, JHEP 9812 (1998) 027 [hep$\mathrm{ph} / 9810442]$.

[8] J. A. Bagger, T. Moroi and E. Poppitz, JHEP 0004 (2000) 009 [hep-th/9911029].

[9] I. Antoniadis and T. Maillard, "Moduli stabilization from magnetic fluxes in type I string theory," Nucl. Phys. B 716 (2005) 3 [hep-th/0412008]; I. Antoniadis, A. Kumar and T. Maillard, "Magnetic fluxes and moduli stabilization," Nucl. Phys. B 767 (2007) 139 [hep-th/0610246].

[10] I. Antoniadis, J.-P. Derendinger and T. Maillard, "Nonlinear N=2 Supersymmetry, Effective Actions and Moduli Stabilization," Nucl. Phys. B 808 (2009) 53 [arXiv:0804.1738 [hep-th]].

[11] I. Antoniadis, A. Chatrabhuti, H. Isono and R. Knoops, "Inflation from Supergravity with Gauged R-symmetry in de Sitter Vacuum,” Eur. Phys. J. C 76 (2016) no.12, 680 [arXiv:1608.02121 [hep-ph]].

[12] A. H. Guth, "The Inflationary Universe: A Possible Solution to the Horizon and Flatness Problems," Phys. Rev. D 23 (1981) 347;

A. D. Linde, "A New Inflationary Universe Scenario: A Possible Solution of the Horizon, Flatness, Homogeneity, Isotropy and Primordial Monopole Problems," Phys. Lett. 108B (1982) 389; A. Albrecht and P. J. Steinhardt, "Cosmology for Grand Unified Theories with Radiatively Induced Symmetry Breaking,” Phys. Rev. Lett. 48 (1982) 1220.

[13] D. H. Lyth and A. Riotto, "Particle physics models of inflation and the cosmological density perturbation," Phys. Rept. 314 (1999) 1 [hep-ph/9807278];

A. D. Linde, "Particle physics and inflationary cosmology," Contemp. Concepts Phys. 5 (1990) 1 [hep-th/0503203].

[14] A. A. Starobinsky,“A New Type of Isotropic Cosmological Models Without Singularity,” Phys. Lett. $91 B$ (1980) 99.

[15] L. Randall and S. D. Thomas, "Solving the cosmological moduli problem with weak scale inflation,” Nucl. Phys. B 449 (1995) 229 [hep-ph/9407248];

A. Riotto, "Inflation and the nature of supersymmetry breaking," Nucl. Phys. B 515 (1998) 413 [hep-ph/9707330]:

K. I. Izawa, "Supersymmetry - breaking models of inflation," Prog. Theor. Phys. 99 (1998) 157 [hep-ph/9708315];

W. Buchmuller, L. Covi and D. Delepine, "Inflation and supersymmetry breaking," Phys. Lett. B 491 (2000) 183 [hep-ph/0006168].

[16] I. Antoniadis, A. Chatrabhuti, H. Isono and R. Knoops, "Inflation from Supersymmetry Breaking," Eur. Phys. J. C 77 (2017) no.11, 724 [arXiv:1706.04133 [hep-th]].

[17] D. Z. Freedman and A. Van Proeyen, Cambridge, UK: Cambridge Univ. Pr. (2012) 607 p.

[18] P. Fayet and J. Iliopoulos, Phys. Lett. B 51 (1974) 461;

P. Fayet, Phys. Lett. B 69 (1977) 489.

[19] I. Antoniadis, J. -P. Derendinger and T. Maillard, Nucl. Phys. B 808 (2009) 53 [arXiv:0804.1738 [hep-th]].

[20] J. Polonyi, Hungary Central Inst Res - KFKI-77-93 (77,REC.JUL 78) 5p.

[21] H. P. Nilles, Phys. Rept. 110 (1984) 1,

[22] S. Ferrara, L. Girardello, T. Kugo and A. Van Proeyen, Nucl. Phys. B 223 (1983) 191,

[23] J. R. Ellis, K. A. Olive, Y. Santoso and V. C. Spanos, Phys. Lett. B 573 (2003) 162 [hep$\mathrm{ph} / 0305212]$,

[24] T. Gherghetta, G. F. Giudice and J. D. Wells, Nucl. Phys. B 559 (1999) 27 [hep-ph/9904378]. 
[25] P. A. R. Ade et al. [Planck Collaboration], "Planck 2015 results. XX. Constraints on inflation," arXiv:1502.02114 [astro-ph.CO].

[26] D. Baumann and D. Green, "Signatures of Supersymmetry from the Early Universe," Phys. Rev. D 85 (2012) 103520 [arXiv:1109.0292 [hep-th]].

[27] K. Schmitz and T. T. Yanagida, "Dynamical supersymmetry breaking and late-time R symmetry breaking as the origin of cosmic inflation," Phys. Rev. D 94 (2016) no.7, 074021 [arXiv:1604.04911 [hep-ph]].

[28] P. Binetruy and G. R. Dvali, “D term inflation,” Phys. Lett. B 388 (1996) 241 [hep-ph/9606342].

[29] C. Wieck and M. W. Winkler, "Inflation with Fayet-Iliopoulos Terms," Phys. Rev. D 90 (2014) no.10, 103507 [arXiv:1408.2826 [hep-th]];

V. Domcke and K. Schmitz, “Unified model of D-term inflation,” Phys. Rev. D 95 (2017) no.7, 075020 [arXiv:1702.02173 [hep-ph]].

[30] D. V. Volkov and V. P. Akulov, Is the neutrino a Goldstone particle?; Phys. Lett. B 46 (1973) 109 ;

M. Roček, Linearizing the Volkov-Akulov model, Phys. Rev. Lett. 41 (1978) 451;

U. Lindström, M. Roček, Constrained local superfields, Phys. Rev. D 19 (1979) 2300;

R. Casalbuoni, S. De Curtis, D. Dominici, F. Feruglio and R. Gatto, Nonlinear realization of supersymmetry algebra from supersymmetric constraint, Phys. Lett. B 220 (1989) 569;

Z. Komargodski and N. Seiberg, From linear SUSY to constrained superfields, JHEP 0909 (2009) 066; [arXiv:0907.2441 [hep-th]];

S. M. Kuzenko and S. J. Tyler, On the Goldstino actions and their symmetries, JHEP 1105, (2011) 055: [arXiv:1102.3043 [hep-th]].

[31] L. Alvarez-Gaume, C. Gomez and R. Jimenez, "Minimal Inflation,” Phys. Lett. B 690 (2010) 68 [arXiv:1001.0010 [hep-th]];

L. Alvarez-Gaume, C. Gomez and R. Jimenez, "A Minimal Inflation Scenario," JCAP 1103 (2011) 027 [arXiv:1101.4948 [hep-th]];

S. Ferrara and D. Roest, "General sGoldstino Inflation," JCAP 1610 (2016) no.10, 038 [arXiv:1608.03709 [hep-th]].

[32] E. J. Copeland, A. R. Liddle, D. H. Lyth, E. D. Stewart and D. Wands, "False vacuum inflation with Einstein gravity," Phys. Rev. D 49 (1994) 6410 [astro-ph/9401011].

[33] D. Baumann and L. McAllister, "Inflation and String Theory," arXiv:1404.2601 [hep-th]; M. Cicoli and F. Quevedo, "String moduli inflation: An overview," Class. Quant. Grav. 28 (2011) 204001 [arXiv:1108.2659 [hep-th]].

[34] G. R. Dvali, Q. Shafi and R. K. Schaefer, "Large scale structure and supersymmetric inflation without fine tuning," Phys. Rev. Lett. 73 (1994) 1886 [hep-ph/9406319].

[35] L. Boubekeur and D. H. Lyth, "Hilltop inflation,” JCAP 0507 (2005) 010 [hep-ph/0502047]. 